\title{
Continuous functions and potential theory
}

\author{
By Hans Wallin
}

\section{Introduction}

Let $F_{0}$ be a compact set in $R^{m}$. If $F_{0}$ has $\alpha$-capacity zero, $0 \leqslant \alpha<m, 1$ there exists, according to a well-known result by Evans [8], a positive measure $\mu$ concentrated on $F_{0}$ such that the potential of order $\alpha$ of $\mu$ is infinite everywhere on $F_{0}$. In Chapter I we consider a problem related to this result. We shall prove that a compact set $F_{0}$ has $\alpha$-capacity zero if and only if every continuous function coincides everywhere on $F_{0}$ with a continuous $\alpha$-potential of a measure with compact support. This is a consequence of the Theorems 1 and 2. However, these theorems contain much more than the above characterization of compact sets of $\alpha$-capacity zero. In particular we consider the case when we have more general kernels than $r^{-\alpha}$.

Let $h$ be a positive integer, $p \geqslant 1,0 \leqslant \alpha<m$, and let $F_{0}$ be a compact set with $\alpha$ capacity zero. In Chapter II we use Theorem 1 to deduce conditions on $h, p$ and $\alpha$ which guarantee that every function $f_{0}$ which is the restiction to $F_{0}$ of a continuous function can be extended to a function $f$ having the following properties: $f$ is defined and continuous everywhere in $R^{m}$ and infinitely differentiable on the complement of $F_{0}$; all the partial derivatives of $f$ of orders less than or equal to $h$ and the function $f$ itself belong to $L^{p}\left(R^{m}\right)$. The result is stated in Theorem 3 . The conditions of the theorem imply in particular $\alpha<m-1$; i.e. all the compact sets considered in Theorem 3 have Hausdorff dimension less than $m-1$.

We formulate a converse of Theorem 3 in $\S 7$ (Theorem 4) where we also consider a certain class of Beppo Levi functions, which, in the case $\alpha<m-1$, is more general than the class of functions considered in Theorem 3 . The case $p=1$ is studied further in $\S 9$.

As a by-product of our investigation we obtain a theorem on the existence of uniformly continuous harmonic functions with finite Dirichlet integrals in the unit sphere, which take given continuous values on a certain subset of the boundary of the unit sphere (Theorem 5).

I wish to thank Professor L. Carleson who suggested the subject of this paper and contributed with many ideas to the proofs of the theorems.

\section{Chapter I. On the representation of continuous functions by potentials}

\section{Notations and definitions}

$R^{m}$ is the $m$-dimensional Euclidean space, $m \geqslant 1$, with points $x=\left(x^{1}, \ldots, x^{m}\right),|x|$ the distance from $x$ to the origin. By a closed cube in $R^{m}$ we mean the set of points satisfying the inequalities $a_{i} \leqslant x^{i} \leqslant a_{i}+l$ where $a_{i}, i=1, \ldots, m$, are any numbers and $l>0$.

$1 \alpha=0$ corresponds to the logarithmic capacity. 
$S\left(x_{0}, r\right)$ denotes the closed sphere $\left|x-x_{0}\right| \leqslant r$. We write $\min \{a, b\}$ for the smaller of the numbers $a$ and $b$.

The complement of a set $E$ we denote by $G E$. If $E_{1}$ and $E_{2}$ are two sets, then $E_{1} \backslash E_{2}$ is the set of points belonging to $E_{1}$ but not to $E_{2}$. If $E_{1} \supset E_{2}$, we write $E_{1}-E_{2}$ instead of $E_{1} \backslash E_{2}$.

By a kernel we mean a function $K$ satisfying the following conditions:

(a) $K$ is defined in the interval $r>0$, is finite and continuous, non-negative and nonincreasing and satisfies $\lim _{r \rightarrow 0} K(r)=\infty$.

$$
\int_{0}^{1} K(r) r^{m-1} d r<\infty .
$$

(a) and (b) are for instance satisfied if $K(r)=r^{-\alpha}, 0<\alpha<m$.

Let $\sigma$ be a real measure on $R^{m}$, i.e. a completely additive real set function. The support of $\sigma$ is denoted by $S_{\sigma} . \sigma^{+}$is the positive and $\sigma^{-}$the negative part of $\sigma, \sigma=$ $\sigma^{+}-\sigma^{-}$, and $|\sigma|=\sigma^{+}+\sigma^{-}$.

The potential of a measure $\sigma$ belonging to a kernel $K$, the $K$-potential of $\sigma$, is denoted by $u_{K}^{\sigma}$,

$$
u_{K}^{\sigma}(x)=\int K(|x-y|) d \sigma(y),{ }^{1}
$$

and the energy integral by $I_{K}(\sigma)$,

$$
I_{K}(\sigma)=\iint K(|x-y|) d \sigma(x) d \sigma(y)
$$

$u_{K}^{\sigma}$ is well-defined at the point $x$ provided $u_{K}^{\sigma+}(x)$ and $u_{K}^{\sigma-}(x)$ are not both infinite. If there will be no misunderstanding we write $u^{\sigma}$ instead of $u_{K}^{\sigma}$. If $\sigma$ is absolutely continuous and has a density $g, d \sigma=g d x$, we sometimes write $u_{K}^{g}$ instead of $u_{K}^{\sigma}$.

The $K$-capacity of a bounded Borel set $E, C_{K}(E)$, is defined as

$$
C_{K}(E)=\left\{\inf _{\nu \in \Gamma E} I_{K}(\nu)\right\}^{-1}
$$

where $\Gamma_{E}$ is the class of positive measures $\nu$ with total mass 1 and $S_{\nu} \subset E$. The $K$ capacity is an inner measure, i.e.

$$
C_{K}(E)=\sup _{F \subset E} C_{K}(F)
$$

where $F$ is a closed subset of $E$.

The $\alpha$-potential and the logarithmic potential, i.e. the potentials in the cases $K(r)=r^{-\alpha}, 0<\alpha<m$, and $K(r)=-\log r,{ }^{2}$ we also denote by $u_{\alpha}^{\sigma}$ and $u_{0}^{\sigma}$ respectively and analogously for the $\alpha$-capacity and the logarithmic capacity.

1 The integration is to be extended over the whole space if no limits of integration are indicated.

2 We shall also consider the case when $K(r)=-\log r$ in spite of the fact that $-\log r$ takes negative values too. We sometimes omit the special simple treatment which is needed in the case $K(r)=-\log r$. 
Our kernel $K$ satisfies the continuity principle (see for instance Ugaheri [18]): If $\mu$ is a positive measure with compact support and the restriction of $u_{K}^{\mu}$ to $S_{\mu}$. is continuous, then $u_{K}^{\mu}$ is continuous in the whole space.

Let $F$ be a compact set with positive $K$-capacity. Then there exists a positive measure $\tau$-which is not necessarily uniquely determined-with $\tau\left(R^{m}\right)=1, S_{\tau} \subset F$, such that

$$
I_{K}(\tau)=\inf _{\nu \in \Gamma_{F}} I_{K}(\nu)=\left\{C_{K}(F)\right\}^{-1},
$$

where $\Gamma_{F}$ is the class of positive measures $\nu$ with $\nu\left(R^{m}\right)=1, S_{v} \subset F$. We call $\tau$ a capacitary distribution and $u_{K}^{\tau}$ a capacitary potential belonging to $K$ and $F . u_{K}^{\tau}$ satisfies the following inequalities:

$u_{K}^{\tau}(x) \geqslant\left\{C_{K}(F)\right\}^{-1} \quad$ for every $x \in F$ except when $x$ belongs to a set of $K$-capacity zero.

$$
u_{K}^{\tau}(x) \leqslant\left\{C_{K}\left(F^{\prime}\right)\right\}^{-1} \quad \text { for every } x \in S_{\tau}
$$

$u_{K}^{\tau}(x) \leqslant A \cdot\left\{C_{K}(F)\right\}^{-1}$ everywhere, where $A$ is a constant which only depends on the dimension $m$ of the space $R^{m}$.

As to (1.2) and (1.3) we refer to Frostman [9, pp. 35 ff.] and Fuglede [11, p. 159]. (1.4) is a result by Ugaheri [18].

If $F_{0}$ is a compact set, we denote by $S\left(F_{0}\right)$ the class of functions which are restrictions to $F_{0}$ of real functions defined and continuous everywhere on $R^{m}$.

\section{Representation of continuous functions by potentials}

In this section we also make the following assumption on our kernel $K$ :

(c) If $E$ is the union of a finite number of closed spheres and $u_{K}^{\tau}$ a capacitary potential belonging to $K$ and $E$, we have

$$
u_{K}^{\tau}(x) \geqslant\left\{C_{K}(E)\right\}^{-1}, \text { for every } x \in E .
$$

The condition (c) is for instance satisfied if $K(r)=r^{-\alpha}, 0<\alpha<m$, and, more generally if $K$ satisfies

where $M$ is a constant. ${ }^{1}$

$$
K(r)<M K(2 r), \text { for every } r>0,
$$

We shall now prove the following theorem on the representation of continuous functions by potentials.

Theorem 1. Suppose that $K$ is a kernel satisfying (c) and that $F_{0}$ is a compact set with $C_{K}\left(F_{0}\right)=0$. Then there exists, for every function $f_{0} \in S\left(F_{0}\right)$, an absolutely continuous measure $\sigma$ with compact support having the following properties: The potential of $\sigma$, $u_{K}^{a}$, is continuous in the whole space and equal to $f_{0}$ on $F_{0}$, i,e.

$$
u_{K}^{\sigma}(x)=f_{0}(x), \quad \text { for every } x \in F_{0} ;
$$

\footnotetext{
1 For a proof of this we refer to Kunugui [13] or Carleson [4, p. 16]. These authors have made further assumptions on the kernel $K$. However, it is easy to see that the result is true also for our kernels.
} 
if $f_{0}(x)>0$ for every $x \in F_{0}$, then $\sigma$ is a positive measure and if $K$ is infinitely differentiable in the interval $r>0$ then $u_{K}^{\sigma}$ is infinitely differentiable on $\mathbf{C} F_{\mathbf{0}}$.

For the proof we need the following simple lemma.

Lemma 1. Let $K$ be a kernel satisfying (c) and $F_{0}$ a compact set with $C_{K}\left(F_{0}\right)=0$. Suppose that $a$ is given, $a>0$. Then there is a set $E$ which is the union of a finite number of closed spheres, $E \supset F_{0}$, so that $C_{K}(E)<a$. If $u_{K}^{\tau}$ is a capacitary potential belonging to $K$ and $E$, then $u_{K}^{\tau}$ is continuous everywhere and if $A$ is the constant in (1.4) we have

$$
\left\{C_{K}(E)\right\}^{-1} \leqslant u_{K}^{\tau}(x) \leqslant A\left\{C_{K}(E)\right\}^{-1} \quad \text { for every } x \in E
$$

and thus in particular for every $x \in F$.

Proof of Lemma 1. We first observe that the existence of a set $E$ follows immediately from the facts that $F_{0}$ is compact and $C_{K}\left(F_{0}\right)=0$. (2.2) is a consequence of (2.1) and (1.4). Using (1.3) we also find that the restriction of $u_{K}^{\tau}$ to $S_{\tau}$ is constant and thus $u_{K}^{\tau}$ is continuous everywhere according to the continuity principle.

Proof of Theorem 1. We first suppose that $f_{0}(x)>0$ for every $x \in F_{0}$. Let $f$ be a continuous extension of $f_{0}$ to $R^{m}$ and $F$ a compact set such that every point of $F_{0}$ is an interior point of $F$ and $f(x)>0$ for every $x \in F$.

We start by proving that for any $\varepsilon>0$ there exists a positive measure $\nu$ with $\nu\left(R^{m}\right)$ less than a given positive number so that $S_{v}$ is a subset of a given neighborhood of $F_{0}$ and $u^{\nu}=u_{K}^{\nu}$ is continuous and satisfies the following inequalities, if $M=3^{m} A+1$, $m$ is the dimension of the space and $A$ the constant in (1.4),

$$
\begin{gathered}
u^{\nu}(x)<f(x) \text { for every } x \in F, \\
u^{y}(x) \geqslant f_{0}(x)-M \varepsilon \text { for every } x \in F_{0} .
\end{gathered}
$$

We introduce the sequence of nets $n=\left\{n_{i}\right\}$, where $n_{0}$ consists of all closed cubes with corners having integer coordinates, and $n_{i}, i>0$, consists of all closed cubes which we obtain by dividing the cubes in $n_{t-1}$ into $2^{m}$ equal cubes by $(m-1)$-dimensional hyperplanes parallel to the coordinate planes. Let $\omega_{1}, \ldots, \omega_{r}$ be a number of congruent cubes from $n, \cup \omega_{i} \supset F$, so that the oscillation of $f$ is less than $\varepsilon$ in $\omega_{i}$ for $i=1, \ldots, r$. We separate those cubes $\omega_{i}, i=1, \ldots, r$, which are such that the maximum of $f_{0}$ on $F_{0} \cap \omega_{i}$ is larger than $M \varepsilon{ }^{1}{ }^{1}$ In this way we get the cubes $\omega_{1}^{\prime}, \ldots, \omega_{s}^{\prime}$. Using Lemma 1 it is easy to realize that, for $i=1, \ldots, s$, we can choose a positive measure with total mass less than a given positive number having the following properties: The support of the measure is a subset of a given neighborhood of $F_{0} \cap \omega_{i}^{\prime}$; the $K$-potential of the measure is continuous everywhere and takes values between $\varepsilon$ and $A \varepsilon$ on $F_{0} \cap \omega_{i}^{\prime}$; it is less than or equal to $A \varepsilon$ on $\omega_{i}^{\prime}$ and on those cubes $\omega_{1}, \ldots, \omega_{r}$ which have non-void intersections with $\omega_{i}^{\prime}$ and it is finally less than a given positive number elsewhere. In this way we get a positive measure associated with every cube $\omega_{i}^{\prime}, i=1, \ldots, s$. Let $\nu_{1}$ be the sum of these measures. Due to the choice of the constant $M, M=3^{m} A+1$, and the fact that the oscillation of $f$ is less than $\varepsilon$ in every cube $\omega_{i}, i=1, \ldots, r$, it is easy to realize that we can make the above procedure so that

$$
\begin{gathered}
u^{\nu_{1}}(x)<f(x) \quad \text { for every } x \in F, \\
u^{\nu_{1}}(x) \geqslant \min \left\{f_{0}(x)-M \varepsilon, \varepsilon\right\} \quad \text { for every } x \in F_{0} .
\end{gathered}
$$

1 If $f_{0}(x) \leqslant M \varepsilon$ for every $x \in F_{0}$ there are no such cubes. In this case, however, (2.3) and (2.4) are satisfied with $\boldsymbol{v}=\mathbf{0}$. 
Our procedure also guarantees that we can get $\nu_{1}\left(R^{m}\right)$ smaller than a given positive number and $S_{v_{1}}$ as a subset of a given neighborhood of $F_{0}$.

If (2.3) and (2.4) are not true for $\nu=\nu_{1}$ we consider $f-u^{\nu_{1}}$. As $f(x)-u^{\nu_{1}}(x)>0$ for every $x \in F$ and $u^{v_{1}}$ is continuous we can repeat the procedure leading to (2.5) and (2.6) but with $f$ replaced by $f-u^{\nu_{1}}$ and $f_{0}$ by $f_{0}-u^{\nu_{1}}$. In this way we get a positive measure $\nu_{2}$ having properties which are analogous to those of $\nu_{1}$, and a continuous potential $u^{\nu_{2}}$ satisfying

$$
\begin{gathered}
u^{\nu_{2}}(x)<f(x)-u^{\nu_{1}}(x) \quad \text { for every } x \in F, \\
u^{v_{2}}(x) \geqslant \min \left\{f_{0}(x)-u^{\nu_{1}}(x)-M \varepsilon, \varepsilon\right\} \text { for every } x \in F_{0} .
\end{gathered}
$$

(2.6) and (2.8) give, for $x \in F_{0}$,

$$
u^{y_{1}}(x)+u^{v_{z}}(x) \geqslant \min \left\{f_{0}(x)-M \varepsilon, 2 \varepsilon\right\}
$$

and so (2.7) and (2.8) yield

$$
\begin{gathered}
u^{\nu_{1}}(x)+u^{\nu_{2}}(x)<f(x) \quad \text { for every } x \in F, \\
u^{\nu_{1}}(x)+u^{\nu_{2}}(x) \geqslant \min \left\{f_{0}(x)-M \varepsilon, 2 \varepsilon\right\} \text { for every } x \in F_{0} .
\end{gathered}
$$

If (2.3) and (2.4) are not true for $\nu=\nu_{1}+\nu_{2}$ we repeat our procedure anew. After $n$ steps we have obtained $n$ positive measures $\nu_{1}, \ldots, v_{n}$ having continuous potentials $u^{\nu_{1}}, \ldots, u^{\nu_{n}}$ so that

$$
\begin{gathered}
\sum_{i=1}^{n} u^{v_{i}}(x)<f(x) \quad \text { for every } x \in F, \\
\sum_{i=1}^{n} u^{\eta_{i}}(x) \geqslant \min \left\{f_{0}(x)-M \varepsilon, n \varepsilon\right\} \quad \text { for every } x \in F_{0},
\end{gathered}
$$

and so that $\sum_{1}^{n} \nu_{i}\left(R^{m}\right)$ is less than a given positive number and $\bigcup_{1}^{n} S_{\nu_{i}}$ is a subset of a given neighborhood of $F_{0}$. As $f_{0}$ is bounded on $F_{0}$, there exists, according to (2.10), a smallest number $n_{0}$ so that (2.4) holds with $\nu=\nu_{1}+\ldots+v_{n_{0}}$. From (2.9) we see that also (2.3) is true for this choice of $\nu$.

As the second step of the proof we show that there even exists an absolutely continuous positive measure, $\psi d x$, where $\psi$ is infinitely differentiable and the total mass of $\psi d x$ is less than a given positive number and $S_{\psi}$ is a subset of a given neighborhood of $F_{0}^{\prime}$, so that (2.3) and (2.4) are true with $\nu$ replaced by $\psi d x$. In fact, let $\varphi$ be an infinitely differentiable function with compact support, $\varphi \geqslant 0, \int \varphi(x) d x=1$, and let $\psi$ be the convolution of $\varphi$ and $\nu, \psi=\varphi * v . \psi$ is then infinitely differentiable and the measure $\psi d x$ has the same total mass as $\nu$. By choosing $r$ small enough and $\varphi$ such that $S_{\varphi} \subset S(0, r)$, where $S(0, r)$ is the sphere with centre 0 and radius $r$, we can make $S_{\psi}$ a subset of a given neighborhood of $S_{v}$. As $u^{\nu}=K * \nu$ we have

$$
u^{\varphi}=K * \psi=K * \varphi * \nu=\varphi * u^{\nu} .
$$

By choosing $r$ small enough we can thus also make the difference $u^{\varphi}-u^{v}$ less than a given number, uniformly on $F$. As we can prove an inequality (2.4) for every $\varepsilon>0$ we conclude that we also can prove the same inequality and the inequality (2.3) with $\nu$ replaced by a measure $\psi d x$ having the properties stated above. 


\section{H. WALLIN, Continuous functions and potential theory}

Thus we have proved the existence of an absolutely continuous measure $\mu_{1}$ having an infinitely differentiable density so that

$$
\begin{array}{lll}
u^{\mu_{1}}(x)<f(x) & \text { for every } & x \in F, \\
u^{\mu_{1}}(x) \geqslant f_{0}(x)-2^{-1} & \text { for every } & x \in F_{0} .
\end{array}
$$

By considering $f-u^{\mu_{1}}$ instead of $f$ we get analogously a measure $\mu_{2}$ so that

$$
\begin{array}{lll}
u^{\mu_{2}}(x)<f(x)-u^{\mu_{1}}(x) & \text { for every } & x \in F^{\prime}, \\
u^{\mu_{2}}(x) \geqslant f_{0}(x)-u^{\mu_{1}}(x)-2^{-2} & \text { for every } & x \in F_{0}, \\
u^{\mu_{1}}(x)+u^{\mu_{2}}(x)<f(x) & \text { for every } & x \in F, \\
u^{\mu_{1}}(x)+u^{\mu_{2}}(x) \geqslant f_{0}(x)-2^{-2} & \text { for every } & x \in F_{0} .
\end{array}
$$

which gives $\quad u^{\mu_{1}}(x)+u^{\mu_{2}}(x)<f(x) \quad$ for every $\quad x \in F$,

After $n$ steps we have obtained $n$ positive measures, $\mu_{1}, \ldots, \mu_{n}$, satisfying

$$
\begin{aligned}
& \sum_{i=1}^{n} u^{\mu_{i}}(x)<f(x) \quad \text { for every } \quad x \in F, \\
& \sum_{i=1}^{n} u^{\mu_{i}}(x) \geqslant f_{0}(x)-2^{-n} \quad \text { for every } \quad x \in F_{0} .
\end{aligned}
$$

We can furthermore suppose that $\mu_{i}$ is absolutely continuous and has an infinitely differentiable density for $i=1, \ldots, n$, that $\sum_{1}^{n} \mu_{i}\left(R^{m}\right)$ is less than a given positive number which is independent of $n$, and that the maximal distance between $F_{0}$ and a point on the boundary of $S_{\mu_{n}}$ tends to zero as $n$ tends to infinity. Under these assumptions it follows that $\left\{\sum_{1}^{n} \mu_{i}\right\}_{n=1}^{\infty}$ converges weakly to an absolutely continuous positive measure $\mu$ such that the density of $\mu$ is infinitely differentiable om $C F_{0}$. Using (2.12) we obtain, as the kernel $K$ is non-negative,

$$
u^{\mu}(x) \geqslant \sum_{1}^{n} u^{\mu_{i}}(x) \geqslant f_{0}(x)-2^{-n}, n=1,2, \ldots, \text { for every } \quad x \in F_{0} .
$$

This gives $u^{\mu}(x) \geqslant f_{0}(x)$ for every $u \in F_{0}$. As $\left\{\sum_{1}^{n} \mu_{i}\right\}$ converges weakly to $\mu$ we have by (2.11):

$$
f(x) \geqslant \lim _{n \rightarrow \infty} \sum_{1}^{n} u^{\mu_{i}}(x) \geqslant u^{\mu}(x) \quad \text { for every } \quad x \in F
$$

It follows that

$$
\begin{gathered}
u^{\mu}(x)=f_{0}(x) \quad \text { for every } \quad x \in F_{0}, \\
u^{\mu}(x) \leqslant f(x) \quad \text { for every } x \in F .
\end{gathered}
$$

From the construction we conclude at once that $u^{\mu}$ is continuous on $\mathbf{C} F_{\mathbf{0}}$. To prove that $u^{\mu}$ is continuous at a point $x_{0}$ belonging to $F_{0}$ we use (2.14). It is well known that

$$
u^{\mu}\left(x_{0}\right) \leqslant \lim _{x \rightarrow x_{0}} u^{\mu}(x)
$$


To prove that

$$
u^{\mu}\left(x_{0}\right) \geqslant \varlimsup_{x \rightarrow x_{0}} u^{\mu}(x)
$$

we observe that $x_{0}$ is an interior point of $F$ and so (2.14) yields

$$
\varlimsup_{x \rightarrow x_{0}} u^{\mu}(x) \leqslant \varlimsup_{x \rightarrow x_{0}} f(x)=f\left(x_{0}\right)=f_{0}\left(x_{0}\right)=u^{\mu}\left(x_{0}\right) .
$$

This proves that $u^{\mu}$ is continuous at $x_{0}$ and thus everywhere.

From the construction of $u^{\mu}$ we easily realize that $u^{\mu}$ is infinitely differentiable on $\mathbf{C} F_{0}$ in the case when $K$ is infinitely differentiable in the interval $r>0$. Because if $x_{1}$ is a point in $\mathrm{G} F_{0}$ we can write $u^{\mu}$ :

$$
u^{\mu}=\sum_{1}^{n_{1}} u^{\mu_{i}}+\left(u^{\mu}-\sum_{1}^{n_{1}} u^{\mu_{i}}\right)
$$

and choose $n_{1}$ so large that the support of $\mu-\sum_{1}^{n_{1}} \mu_{i}$ does not contain $x_{1}$. The second term of the right member of (2.15) is then infinitely differentiable at $x_{1}$ due to our assumption on $K$, and the first term due to the fact that $\sum_{1}^{n_{1}} \mu_{i}$ is absolutely continuous and has an infinitely differentiable density.

By that our theorem is completely proved in the case when $f_{0}(x)>0$ for every $x \in F_{0}$. The general case follows immediately by writing $f_{0}$ as the difference between two functions which are strictly positive on $F_{0}$.

Remark 1. From the proof it is clear that the measure $\sigma$ in Theorem 1 can be chosen with $\sigma^{+}\left(R^{m}\right)+\sigma^{-}\left(R^{m}\right)$ less than a given number so that $S_{\sigma}$ is a subset of a given neighborhood of $F_{0}$. We can also make $u^{\sigma}$ the difference between two continuous potentials generated by absolutely continuous positive measures having densities which are infinitely differentiable on $\mathrm{CF}_{\mathbf{0}}$.

Remark 2. The condition $K$ non-negative is not necessary for the validity of Theorem 1. For instance, it is easy to realize that the theorem is true also for the kernel $-\log r$.

Remark 3. From Theorem 1 we can deduce the following result: Suppose that $g$ is a strictly positive function which is lower semi-continuous in $R^{m}$ and that $C_{K}\left(F_{0}\right)=0$, $F_{0}$ compact. Then there exists a potential $u_{K}^{\mu}$, continuous on $\mathbf{C} F_{0}$, which is generated by a positive absolutely continuous measure $\mu$ such that $S_{\mu}$ is a subset of a given neighborhood of $F_{0}$ and

$$
u^{\mu}(x)=g(x) \quad \text { for every } \quad x \in F_{\mathbf{0}} .
$$

In fact, there exists a sequence of continuous functions $\left\{g_{i}\right\}_{1}^{\infty}, 0<g_{i-1}(x)<g_{i}(x)$ for every $x \in F_{0}, i=2,3, \ldots$, converging pointwise to $g$ on $F_{0}$. Hence there is a positive measure $\mu_{i}$ having suitable properties and a continuous potential $u^{\mu_{i}}$ so that, if $g_{0}=0$,

$$
u^{\mu_{i}}(x)=g_{i}(x)-g_{t-1}(x) \quad \text { for every } \quad x \in F_{0}, i=1,2, \ldots
$$

It is possible to arrange so that $\left\{\sum_{1}^{n} \mu_{i}\right\}_{1}^{\infty}$ converges weakly to a positive measure $\mu$ having the required properties and in fact also those stated for $\sigma$ in Remark 1 . This gives the required properties to $u^{\mu}$ including the equality $u^{\mu}(x)=g(x)$ for every $x \in F_{0}$. (Compare the calculations leading from (2.11) and (2.12) to (2.13).) 


\section{H. WALLIN, Continuous functions and potential theory}

This result clearly contains the following result by Rudin [16] as a special case (the case $g(x)=\infty$ for every $x \in F_{0}$ ): Suppose that $G$ is an open set containing the compact set $F_{0}$ and that $C_{m-2}\left(F_{0}\right)=0, m \geqslant 2$. Then there exists a positive absolutely continuous measure $\mu$ having compact support such that $u_{m-2}^{\mu}$ is infinite on $F_{0}$, continuous on $\mathrm{CF}_{0}$ and harmonic in the complement of the closure of $G{ }^{1}$

Remark 4. Let $U$ be the open unit sphere in $R^{m}$. If $F_{0}$ is a closed subset of the boundary of $U, f_{0} \in S\left(F_{0}\right)$ and $C_{m-2}\left(F_{0}\right)=0, m \geqslant 2$, it is not hard to prove, by modifications of the proof of Theorem 1 , that there exists a measure $\sigma$ such that $S_{\sigma}$ is compact and does not contain any points from $U$ and such that $u_{m-2}^{\sigma}(x)=f_{0}(x)$ for every $x \in F_{0}$. As before we can get $u_{m-2}^{\sigma}$ as the difference between two continuous potentials generated by positive measures which means that the energy integral $I_{m-2}(|\sigma|)$ is finite. Therefore we can conclude: For any function $f_{0} \in S\left(F_{0}\right)$ there exists a function $u, u(x)=f_{0}(x)$ for every $x \in F_{0}$, which is harmonic in $U$, continuous in the closure of $U$ and has a finite Dirichlet integral,

$$
\int_{U} \sum_{i=1}^{m}\left(\frac{\partial u}{\partial x^{i}}\right)^{2} d x<\infty .
$$

A converse of this proposition will be proved in $\$ 8$.

\section{Modulus of continuity of potentials}

We consider two kernels $K$ and $K_{0}$ and suppose that

$$
\lim _{r \rightarrow 0} K_{0}(r)\{K(r)\}^{-1}=\infty \text {. }
$$

We shall show the existence of a modulus of continuity on the set where $u_{K_{0}}^{|\sigma|}$ is bounded by a given constant, a modulus of continuity which is common to all potentials $u_{K}^{\sigma}$ with $\sigma^{+}\left(R^{m}\right)+\sigma^{-}\left(R^{m}\right)$ less than a given constant. In $\S 4$ we shall then use this result to prove a converse of Theorem 1 .

Lemma 2. Suppose that the kernels $K$ and $K_{0}$ satisfy (3.1). Then there exists a nonnegative function $t$ defined in the interval $r>0, \lim _{r \rightarrow 0} t(r)=0$, only depending on $K$ and $K_{0}$ so that, if $\sigma$ is a measure with compact support, $\sigma^{+}\left(R^{m}\right)+\sigma^{-}\left(R^{m}\right) \leqslant M_{1}$ and $u_{R_{a}}^{|\sigma|}\left(x_{i}\right) \leqslant M_{2}, i=1,2$, then we have

$$
\left|u_{K}^{\sigma}\left(x_{1}\right)-u_{K}^{\sigma}\left(x_{2}\right)\right| \leqslant\left(M_{1}+M_{2}\right) t\left(\left|\dot{x}_{1}-x_{2}\right|\right) .
$$

Proof. We consider only the case when $\sigma$ is a positive measure, from which the general case is an immediate consequence. Let $x_{1}$ and $x_{2}$ be two points with $u_{K_{0}}^{\sigma}\left(x_{i}\right)$ $\leqslant M_{2}, i=1,2$, and put $\left|x_{1}-x_{2}\right|=r_{0}$. Let $S$ be the open sphere with centre $\left(x_{1}+x_{2}\right) \cdot 2^{-1}$ and radius $2^{-1} r_{0}+r_{1}$, where $r_{1}$ is a number which we shall choose later depending on $r_{0}$ so that $r_{1}$ tends to zero when $r_{0}$ tends to zero.

$$
u_{K}^{\sigma}\left(x_{1}\right)-u_{K}^{\sigma}\left(x_{2}\right)=\int\left(K\left(\left|x_{1}-y\right|\right)-K\left(\left|x_{2}-y\right|\right)\right) d \sigma(y)=\int_{S}+\int_{\mathrm{GS}}=I+I I .
$$

1 Rudin also treats the simple extension to arbitrary closed sets. 
In order to estimate $I$ we introduce the function $t_{1}$ :

$$
t_{1}(r)=\left\{K_{0}(r)\right\}^{-1} \cdot K(r) .
$$

$t_{1}$ satisfies $\lim _{r \rightarrow 0} t_{1}(r)=0$ according to (3.1). If $\sigma(x ; r)$ is the value of $\sigma$ for the open sphere with centre $x$ and radius $r$, we have

$$
\begin{aligned}
|I| & \leqslant \int_{0}^{r_{0}+r_{1}} K(r) d \sigma\left(x_{1} ; r\right)+\int_{0}^{r_{0}+r_{1}} K(r) d \sigma\left(x_{2} ; r\right) \\
& =\int_{0}^{r_{0}+r_{1}} t_{1}(r) K_{0}(r) d \sigma\left(x_{1} ; r\right)+\int_{0}^{r_{0}+r_{1}} t_{1}(r) K_{0}(r) d \sigma\left(x_{2} ; r\right) \\
& \leqslant \sup _{0 \leqslant r \leqslant r_{0}+r_{1}} t_{1}(r)\left\{u_{K_{0}}^{\sigma}\left(x_{1}\right)+u_{K_{0}}^{\sigma}\left(x_{2}\right)\right\} \leqslant 2 M_{2} \sup _{0 \leqslant r \leqslant r_{0}+r_{1}} t_{1}(r),
\end{aligned}
$$

i.e.

$$
|I| \leqslant 2 M_{2} \sup _{0 \leqslant r \leqslant r_{0}+r_{1}} t_{1}(r) .
$$

To be able to estimate $I I$ we have to examine the difference $K\left(\left|x_{1}-y\right|\right)-K\left(\left|x_{2}-y\right|\right)$ when $y \in G S$. We need a result of the following kind: There exists a non-negative function $t_{2}$ defined in the interval $r>0, \lim _{r \rightarrow 0} t_{2}(r)=0$, only depending on $K$, so that, for every $\eta>0$,

$$
K(r)-K(r+\varrho) \leqslant t_{2}(\eta) \text { for } \quad r \geqslant t_{2}(\eta), \quad 0 \leqslant \varrho \leqslant \eta .
$$

We suppose for a moment that this has been proved and use it to estimate $I I$. As ||$x_{1}-y|-| x_{2}-y|| \leqslant\left|x_{1}-x_{2}\right|=r_{0}$ and $\left|x_{i}-y\right| \geqslant r_{1}, i=1,2$, when $y \in C S$, we have by using (3.4) with $\eta=r_{0}$ and choosing $r_{1}=t_{2}\left(r_{0}\right)$,

$$
|I I| \leqslant \int_{G S}\left|K\left(\left|x_{1}-y\right|\right)-K\left(\left|x_{2}-y\right|\right)\right| d \sigma(y) \leqslant M_{1} \cdot t_{2}\left(r_{0}\right) \text {. }
$$

We define $t$ by

$$
t\left(r_{0}\right)=2 \sup t_{1}(r)+t_{2}\left(r_{0}\right), \quad r_{0}>0,
$$

where sup is taken for those $r$ which satisfy $0 \leqslant r \leqslant r_{0}+t_{2}\left(r_{0}\right) . t$ satisfies the demands of the lemma; (3.3) and (3.5) give (3.2).

It remains to prove the existence of a function $t_{2}, \lim _{r \rightarrow 0} t_{2}(r)=0$, only depending on $K$, such that (3.4) is valid. We observe that $K$ is uniformly continuous in the interval $a \leqslant r<\infty$ for every $a>0$. From this we conclude that for every $\varepsilon>0$ there exists a largest number $q(\varepsilon)$, which is possibly $+\infty$, so that

$$
K(r)-K(r+\varrho) \leqslant \varepsilon \quad \text { for } \quad r \geqslant \varepsilon, \quad 0 \leqslant \varrho \leqslant q(\varepsilon),
$$

and it is not hard to realize that this implies the existence of a function $t_{2}$ with the desired properties so that (3.4) is true. With that the lemma is proved.

\section{A converse of Theorem 1}

We denote by $U\left(K, F_{0}\right)$ the class of functions which are restrictions to $F_{0}$ of $K$-potentials $u_{K}^{\sigma}$ of measures $\sigma$ with compact supports. A function $f_{0} \in S\left(F_{0}\right)$ belongs to $U\left(K, F_{0}\right)$ if there is a function $g \in \mathcal{U}\left(K, F_{0}\right)$ well-defined at every point of $F_{0}$, 
such that $f_{0}(x)=g(x)$ for every $x \in F_{0}$. We shall prove the following converse of Theorem I:

Theorem 2. Let $K$ be a kernel and $F_{0}$ a compact set, $C_{K}\left(F_{0}\right)>0$. Then there exists a function $f_{0}$ belonging to $S\left(F_{0}\right)$ but not to $\mathcal{U}\left(K, F_{0}\right)$.

Remark 1. From the proof of Theorem 2 it will appear that also the following more general proposition is true: We can find a function $f_{0} \in S\left(F_{0}\right)$ such that there is no function from $U\left(K, F_{0}\right)$ coinciding with $f_{0}$ on $F_{0}$ except on a subset of $F_{0}$ of $K$-capacity zero.

Remark 2. It will appear from the proof that the assumption $K$ non-negative is not necessary for the validity of the theorem. For instance the theorem is true also for the kernel $-\log r$.

Remark 3. Theorem 1 and Theorem 2 give in particular the following characterization of compact sets of $K$-capacity zero: Let $K$ satisfy the condition (c) of $\S 2 . A$ compact set $F_{0}$ has $K$-capacity zero if and only if every function from $S\left(F_{0}\right)$ is the restriction to $F_{0}$ of a continuous $K$-potential of a measure with compact support.

Proof of Theorem 2. As $C_{K}\left(F_{0}\right)>0$ there exists a kernel $K_{0}$ such that

$$
\lim _{r \rightarrow 0} K_{0}(r) \cdot\{K(r)\}^{-1}=\infty
$$

and

$$
C_{K_{0}}\left(F_{0}\right)>0 . .^{1}
$$

According to (4.2) there is a positive measure $\nu_{0}$ with total mass $1, S_{v_{0}} \subset F_{0}$ and $I_{R_{0}}\left(v_{0}\right)<\infty$.

We first use Lemma 2 to deduce a property on $F_{0}$ of an arbitrary $K$-potential $u_{K}^{\sigma}$ of a measure $\sigma$ with compact support and after that we shall construct a continuous function $f$ with restriction $f_{0}$ to $F_{0}$ such that $f_{0}$ does not have this property. Let $\sigma$ be a measure with compact support and $M_{1}=\sigma^{+}\left(R^{m}\right)+\sigma^{-}\left(R^{m}\right)$. As $u_{K_{0}}^{|\sigma|}$ is finite except on a set of $K_{0}$-capacity zero and $v_{0}$, due to the fact that $I_{K_{0}}\left(v_{0}\right)<\infty$, does not concentrate any mass on such a set, we conclude that the set where $u_{K_{0}}^{|\sigma|}$ is finite has $\nu_{0}$-measure 1 , i.e. measure 1 with respect to $\nu_{0}$. Consequently, for every $\varepsilon>0$ there is a constant $M_{2}=M_{2}(\varepsilon)$ so that $u_{K_{0}}^{|\sigma|}$ is less than $M_{2}$ on $F_{0}$ except on a subset of $F_{0}$ having $v_{0}$-measure less than $\varepsilon$. According to (4.1) and Lemma 2 this means that there exists a function $t$ defined in the interval $r>0, \lim _{r \rightarrow 0} t(r)=0$, only depending on $K$ and $K_{0}$ such that

$$
\left|u_{K}^{\sigma}\left(x_{1}\right)-u_{K}^{\sigma}\left(x_{2}\right)\right| \leqslant\left(M_{1}+M_{2}\right) t\left(\left|x_{1}-x_{2}\right|\right),
$$

for $x_{1}$ and $x_{2}$ belonging to $F_{0}$ except when $x_{1}$ and $x_{2}$ belong to a subset of $F_{0}$ having $v_{0}$-measure less than $\varepsilon$.

We construct the function $f$ in the proof of the following lemma:

Lemma 3. Let $K_{0}$ be a kernel, $F_{0}$ a compact set with $C_{K_{0}}\left(F_{0}\right)>0, v_{0}$ a positive measure with $S_{v_{0}} \subset F_{0}, \nu_{0}\left(R^{m}\right)=1, I_{K_{0}}\left(\nu_{0}\right)<\infty$ and $t^{*}$ a non-decreasing function defined in the interval $r>0$ such that $t^{*}(r)>0$ if $r>0, \lim _{r \rightarrow 0} t^{*}(r)=0$. Then there exists a function

1 Compare Carleson [3, p. 405]. 
$f$, defined and continuous everywhere, having the following property for all sufficiently small positive values of $\varepsilon$ : For every Borel set $E, E \subset F_{0}$, with $\nu_{0}(E)<\varepsilon$, there are points $x_{1}$ and $x_{2}$ belonging to $F_{0}-E$ with $\left|x_{1}-x_{2}\right|$ arbitrarily small so that

$$
\left|f\left(x_{1}\right)-f\left(x_{2}\right)\right| \geqslant M_{3} t^{*}\left(\left|x_{1}-x_{2}\right|\right), \quad M_{3} \text { positive constant. } 1
$$

Using Lemma 3 we can easily finish the proof of Theorem 2 . Let $K_{0}, F_{0}$ and $\nu_{0}$ in the lemma be identical with the kernel $K_{0}$, the set $F_{0}$ and the measure $v_{0}$ occurring in the proof of the theorem. We choose the function $t^{*}$ in the lemma so that

$$
\lim _{r \rightarrow 0} t^{*}(r)\{t(r)\}^{-1}=\infty .
$$

If $f$ is the function occurring in the lemma it is clear by (4.3), (4.4) and (4.5) that there is no measure $\sigma$ with compact support such that $u_{K}^{\sigma}$ and $f$ coincide everywhere on $F_{0}$. This proves our theorem.

Proof of Lemma 3. We shall construct $f$ as a sum, $f(x)=\sum_{1}^{\infty} f_{i}(x)$. To construct $\left\{f_{i}\right\}_{1}^{\infty}$ we use three sequences of positive numbers $\left\{a_{i}\right\},\left\{b_{i}\right\}$ and $\left\{d_{i}\right\}$.

We first suppose that the intersection between $F_{0}$ and an arbitrary $(m-1)$-dimensional plane parallel to some coordinate plane has $\nu_{0}$-measure zero.

To construct $f_{i}$, for a fixed $i$, we start by covering $F_{0}$ by means of congruent cubes from the sequence of sets $n=\left\{n_{i}\right\}$ which we used in the proof of Theorem 1 , and we use cubes with diameters less than or equal to $2^{-1} \cdot a_{i}{ }^{2}$ After that we separate those cubes, say $\omega_{i 1}^{\prime}, \omega_{i 2}^{\prime}, \ldots$, which intersect $F_{0}$ in a set of positive $\nu_{0}$-measure. For every cube $\omega_{i}^{\prime}$, where $j$ is a fixed number, $j=1,2, \ldots$, we consider the infinite strip which is determined by the points $\left(x^{1}, \ldots, x^{m}\right)$ where $x^{1}$ varies arbitrarily and $x^{2}, \ldots, x^{m}$ vary in the same intervals as the corresponding coordinates for an arbitrary point in $\omega_{i j}^{\prime}$. If there is no cube $\omega_{i s}^{\prime}, s \neq j$, which is contained in this strip and has an $(m-1)$ dimensional edge plane in common with $\omega_{i j}^{\prime}$, we divide $\omega_{i j}^{\prime}$ into two rectangles by an $(m-1)$-dimensional hyperplane perpendicular to the $x^{1}$-axis so that both rectangles get the property that they intersect $F_{0}$ in a set of positive $y_{0}$-measure. (Compare (4.9) below.) The cubes $\omega_{i \mathrm{i}}^{\prime}, \omega_{i 2}^{\prime}, \ldots$ are in this way replaced by rectangles $\omega_{i \mathrm{1}}^{\prime \prime}, \omega_{i 2}^{\prime \prime}, \ldots$.

We now choose the number $d_{i}$ satisfying

$$
0<d_{i}<\min _{j} v_{0}\left(F_{0} \cap \omega_{i j}^{\prime \prime}\right) .
$$

If $v_{0}\left(F_{0} \cap \omega_{i j}^{\prime \prime}\right)>2 d_{i}$ we divide, for $j=1,2, \ldots, \omega_{i j}^{\prime \prime}$ into two or more parts by $(m-1)$ dimensional hyperplanes perpendicular to the $x^{1}$-axis in such a way that $\omega_{i 1}^{\prime \prime}, \omega_{i 2}^{\prime \prime}, \ldots$ are replaced by rectangles $\omega_{i 1}, \ldots, \omega_{i n_{i}}$ with the following properties:

$$
\begin{gathered}
v_{0}\left(F_{0} \backslash \backslash \bigcup_{j=1}^{n_{i}} \omega_{i j}\right)=0 . \\
d_{i}<v_{0}\left(F_{0} \cap \omega_{i j}\right) \leqslant 2 d_{i}, \quad j=1, \ldots, n_{i} .
\end{gathered}
$$

Every $\omega_{i j}$ has an $(m-1)$-dimensional edge plane in common with a rectangle $\omega_{i s}, s \neq j$, in the infinite strip which is determined by the points $\left(x^{1}, \ldots, x^{m}\right)$ where $x^{1}$ is arbitrary and $x^{2}, \ldots, x^{m}$ vary in the same intervals as the corresponding coordinates for an arbitrary point of $\omega_{i j}$.

\footnotetext{
1 In general we cannot make (4.4) true for all $x_{1}$ and $x_{2}$ belonging to $F_{0}$. This is a consequence of a result by Besicovitch, [1, p. 183].

2 We suppose for the moment that the number $\alpha_{i}$ is given.
} 
As the intersection between $F_{0}$ and an $(m-1)$-dimensional hyperplane parallel to some coordinate plane has $\nu_{0}$-measure zero, we can form a subset $\Delta_{i j}$ of $\omega_{i j}$ containing the boundary of $\omega_{i j}$ such that the distance from the boundary of $\omega_{i j}$ to $\omega_{i j}-\Delta_{i j}$ is larger than zero and, due to (4.8),

$$
\nu_{0}\left(F_{0} \cap\left(\omega_{i j}-\Delta_{i j}\right)\right)>d_{i}, \quad j=1, \ldots, n_{i} .
$$

We can also make the choice of $\Delta_{i j}$ such that, if $\eta$ is a given number, $\eta>0$, and we define $\Delta$ by

$$
\Delta=\bigcup_{i=1}^{\infty} \bigcup_{j=1}^{n_{i}}\left(\Delta_{i j} \cap F_{0}\right)
$$

then we have $\nu_{0}(\Delta)<\eta$.

We now choose $f_{i}$. For every rectangle $\omega_{i j}$ there is an infinite strip of the kind described in (4.9). In each such strip we choose $f_{i}$ identically equal to $t^{*}\left(a_{i}\right)$ in every second set $\omega_{i j}-\Delta_{i j}$ and identically equal to zero in every second, counted from sets $\omega_{i j}-\Delta_{i j}$ with points having smaller $x^{1}$-coordinates to sets with points having larger $x^{1}$-coordinates. In the remaining points of $R^{m}$ we define $f_{i}$ such that $f_{i}$ becomes continuous and less than or equal to $t^{*}\left(a_{i}\right)$ everywhere and zero on $\mathcal{G} \bigcup_{j=1}^{n_{i}} \omega_{i}$.

We now fix two points $x_{i}$ and $x_{i}^{\prime}$ from $\bigcup_{-1}^{n_{i}}\left(\omega_{i j} \cap F_{0}\right) \backslash \Delta$ belonging to two different rectangles $\omega_{i}$ bordering on each other in an infinite strip of the kind described in (4.9). If we introduce the notation $\delta_{j s}^{(i)}$ for the distance between $\omega_{i j}-\Delta_{i j}$ and $\omega_{i s}-\Delta_{i s}$ and define $b_{i}$ by

$$
b_{i}=\min _{j \neq s} \delta_{j s}^{(i)}
$$

then, clearly, we have

$$
\left|f_{i}\left(x_{i}\right)-f_{i}\left(x_{i}^{\prime}\right)\right|=t^{*}\left(a_{i}\right) \text { and } b_{i} \leqslant\left|x_{i}-x_{i}^{\prime}\right| \leqslant a_{i} .
$$

As the function $t^{*}$ is non-decreasing, we obtain

$$
\left|f_{i}\left(x_{i}\right)-f_{i}\left(x_{i}^{\prime}\right)\right| \geqslant t^{*}\left(\left|x_{i}-x_{i}^{\prime}\right|\right) \text {. }
$$

For $j>i$ we have

$$
\left|f_{j}\left(x_{i}\right)-f_{j}\left(x_{i}^{\prime}\right)\right| \leqslant 2 \max _{y}\left|f_{j}(y)\right| \leqslant 2 t^{*}\left(a_{j}\right),
$$

which gives $\quad\left|f_{j}\left(x_{i}\right)-f_{j}\left(x_{i}^{\prime}\right)\right| \leqslant t^{*}\left(\left|x_{i}-x_{i}^{\prime}\right|\right) \cdot 2 t^{*}\left(a_{j}\right) \cdot\left\{t^{*}\left(b_{i}\right)\right\}^{-1}, \quad j>i$.

If we furthermore assume that

$$
a_{i}<b_{i-1}, \quad i=2,3, \ldots,
$$

we get

$$
f_{j}\left(x_{i}\right)-f_{j}\left(x_{i}^{\prime}\right)=0, \quad j<i \text {. }
$$

Putting $f(x)=\sum_{1}^{\infty} f_{1}(x)$ we have by (4.12), (4.13) and (4.15)

$$
\left|f\left(x_{i}\right)-f\left(x_{i}^{\prime}\right)\right| \geqslant t^{*}\left(\left|x_{i}-x_{i}^{\prime}\right|\right)\left[1-2\left\{t^{*}\left(b_{i}\right)\right\}^{-1} \cdot \sum_{j>i} t^{*}\left(a_{j}\right)\right] .
$$

If the expression in square brackets is larger than or equal to $2^{-1}$, i.e. if

$$
\sum_{j>i} t^{*}\left(a_{j}\right) \leqslant \frac{1}{t} t^{*}\left(b_{i}\right)
$$


then

$$
\left|f\left(x_{i}\right)-f\left(x_{i}^{\prime}\right)\right| \geqslant \frac{1}{2} t^{*}\left(\left|x_{i}-x_{i}^{\prime}\right|\right), i=1,2, \ldots
$$

It is easy to realize that it is possible to choose the sequence $\left\{a_{i}\right\}$ such that (4.14) and (4.16) are satisfied and this shows that it is possible to perform our construction. (4.16) guarantees that $\sum f$ converges uniformly and therefore $f$ is continuous.

Suppose now that $E$ is a Borel set, $E \subset F_{0}, v_{0}(E)<\varepsilon$. To finish the proof of the lemma it is, according to (4.17), enough to prove that, for all sufficiently small values of $\varepsilon$ and for all $i$, there exist points $x_{i}$ and $x_{i}^{\prime}$ from $\bigcup_{j=1}^{n_{i}}\left(\omega_{i j} \cap F_{0}\right) \backslash(E \cup \Delta)$ belonging to two different rectangles $\omega_{i j}$ bordering on each other in an infinite strip of the kind described in (4.9). Suppose, on the contrary, that this is not the case. Thus, for some $i$, at least one third of the rectangles $\omega_{i 1}, \ldots, \omega_{i n_{i}}$ do not contain any points from $F_{0} \backslash(E \cup \Delta)$. Using (4.8) and the fact that we have chosen $\Delta$ so that $\nu_{0}(\Delta)<\eta$ we get

$$
\varepsilon+\eta>\nu_{0}(E \cup \Delta)>d_{i} \cdot \frac{n_{i}}{3},
$$

where $n_{i}$ is the number of rectangles $\omega_{i j}$ for a fixed $i$. But (4.7) and (4.8) give

$$
1=\nu_{0}\left(F_{0}\right) \leqslant 2 d_{i} n_{i}
$$

which, combined with (4.18), gives $\varepsilon+\eta>6^{-1}$. As $\eta$ can be chosen arbitrarily small we get a contradiction if $\varepsilon$ is small enough.

The lemma is thus proved in the case when the intersection between $F_{0}$ and an arbitrary $(m-1)$-dimensional hyperplane parallel to some coordinate plane has $v_{0}$-measure zero. If this condition is not satisfied we choose an $(m-l)$-dimensional hyperplane $P$ parallel to some coordinate plane such that $\nu_{0}\left(P \cap F_{0}\right)>0$ and carry through the above construction of $f$-with $F_{0}$ replaced by $P \cap F_{0}$-in the $(m-1)$ dimensional hyperplane $P$. The fact that we may have $v_{0}\left(P \cap F_{0}\right)<1$ does not change the idea of the construction. After having constructed $f$ in $P$ we extend $f$ to a continuous function in $R^{m}$. The extended function clearly satisfies the conditions of the lemma which thus is proved.

\section{Chapter II. An extension problem for continuous functions}

\section{Statement of the problem}

We first introduce some more notations. We denote the sequence $\left(s_{1}, \ldots, s_{h}\right)$ of indices between 1 and $m$ by $s$ and its length $h$ by $|s|$ and we put

$$
D^{s}=\frac{\partial^{h}}{\partial x^{s_{1}} \ldots \partial x^{e_{h}}} .
$$

For any number $p \geqslant 1$ we denote by $L^{p}$ the class of all Lebesgue measurable functions $f$ in $R^{m}$ such that $\int|f(x)|^{p} d x<\infty$; we denote by $L_{\text {loc }}^{p}$ the class of all measurable functions $f$ in $R^{m}$ such that $\int_{F}|f(x)|^{p} d x<\infty$ for every compact set $F$. We use the notation

$$
\|f\|_{L^{p_{(E)}}}=\left\{\int_{E}|f(x)|^{p} d x\right\}^{1 / p}
$$

and we write $\|f\|_{L^{p}}$ instead of $\|f\|_{L^{\left.p_{(} R^{m}\right)}}$. 


\section{H. WALLIN, Continuous functions and potential theory}

We shall use a class of distributions in $R^{m}$ (in the sense of Schwartz [17]) consisting of functions from $L_{\text {loc }}^{1}$ and, in order to avoid confusion with the usual pointwise derivation, we always state explicitly when it concerns derivation in the distribution sense. $\delta$ denotes the Dirac measure and we write $D^{s}$ instead of $\mathrm{D}^{s} \delta$.

We shall deal with the following extension problem: Let $F_{0}$ be a compact set having $m$-dimensional Lebesgue measure zero. We are interested in finding conditions on $F_{0}$ which guarantee that every function $f_{0} \in S\left(F_{0}\right)$ has a continuous extension $f$ to $R^{m}$ such that all the derivatives of $f$-in a certain sense-of orders less than or equal $h$ belong to $L^{p}$, where $h$ and $p$ are given numbers, $p \geqslant 1$. It turns out that a relevant condition is that $C_{\alpha}\left(F_{0}\right)=0$ for a certain $\alpha, 0 \leqslant \alpha<m$, irrespective of the regularity of the set $F_{0}$ in other respects. The results are formulated in the Theorems 3,4 and 6 as conditions on the connections between $h, p, \alpha$ and the dimension $m$ of the space.

In the case when we are searching for a positive solution of our extension problem it is natural to require that the extended function $f$ is to be infinitely differentiable on $\mathbf{C} F_{0}$ (Theorem 3). When we try to find a converse (Theorem 4 ) of Theorem 3 we shall use a certain class of Beppo Levi functions of order $h, B L_{h}\left(L_{\mathrm{loc}}^{p}\right),{ }^{1} p \geqslant 1$. This is the class of distributions $T$ in $R^{m}$ such that all the derivatives (in the distribution sense) of order $h$ are functions belonging to $L_{\mathrm{loc}}^{p}$, i.e. $D^{s} T \in L_{\mathrm{loc}}^{p}$ for all $s$ with $|s|=h$.

We start our investigation by discussing some properties of the class $B L_{h}\left(L_{\mathrm{loc}}^{p}\right)$, $p \geqslant 1$, and the class of functions which are infinitely differentiable on $\mathbf{C} F_{0}$, where $C_{\alpha}\left(F_{0}\right)=0$ for some $\alpha<m-1$ :

$1^{\circ}$. Following Deny-Lions $\left[7\right.$, p. 314] we say that a function $g$, defined in $R^{m}$, has the property $(A C)$ in $R^{m}$ if $g$ is absolutely continuous on almost every line ${ }^{2}$ with a given direction if this direction coincides with the direction of some coordinate axis.

Using the property $(A C)$ we get the following characterization of the distributions in $B L_{h}\left(L_{1 \mathrm{oc}}^{p}\right)$ :

$B L_{h}\left(L_{\text {loc }}^{p}\right)$ consists of those distributions $T$ in $R^{m}$ which have the following properties: Every derivative $D^{s} T$ (in the distribution sense) with $0 \leqslant|s|<h^{3}$ is a function which-properly defined on a set of Lebesgue measure zero-gives a function $g_{s}$ having the property. $(A C)$; all the derivatives of the first order of $g_{s}$ in the usual pointwise sense are in $L_{\mathrm{loc}}^{p}$ and they also constitute the derivatives of $g_{s}$ in the distribution sense.

This characterization is given by Deny and Lions [7, p. 315] for $h=1$. The general case follows easily from the case $h=1$ if we use a theorem by Kryloff [17, part II, p. 37] to conclude that if all the derivatives of the first order of a distribution are functions in $L_{\mathrm{loc}}^{p}$ then the distribution itself is a function in $L_{\mathrm{loc} \cdot{ }^{4}}^{p}$

$2^{\circ}$. $F_{0}$ is a compact set with $C_{\alpha}\left(F_{0}\right)=0$ for some $\alpha$ satisfying $\alpha<m-1$. Suppose that the function $g$ is defined and infinitely differentiable on $\mathrm{G} F_{0}$ and that all the partial derivatives of $g$ of order $h$ belong to $L_{100}^{p}$, where $p \geqslant 1 . C_{\alpha}\left(F_{0}\right)=0$ and $\alpha<m-1$ mean that almost every line with a given direction does not intersect $F_{0}$. Otherwise

\footnotetext{
1 The discussion of this chapter concerning Beppo Levi functions in the case $h=1$, should be compared to the discussions in Deny [5] and Deny-Lions [7]. The definition of $B L_{h}\left(L_{\text {loc }}^{p}\right)$ is found in the work of Deny-Lions. Compare also Nikodym [14].

2 A function is absolutely continuous on a line if the restriction of the function to an arbitrary compact interval of the line is absolutely continuous.

${ }^{3} D^{s} T$ with $|s|=0$ denotes $T$.

4 Observe that by means of Kryloff's theorem it is possible to enunciate more than we have done in our characterization of the distributions in $B L_{h}\left(L_{\text {loc }}^{p}\right)$.
} 
there would exist a direction such that the orthogonal projection $F_{0}^{\prime}$ of $F_{0}$ on an $(m-1)$-dimensional normal plane to this direction would satisfy $C_{\beta}\left(F_{0}^{\prime}\right)>0$ for every $\beta<m-1$, contrary to the fact that $C_{\alpha}\left(F_{0}\right)=0 .{ }^{1}$ This shows that $g$ and all the partial derivatives of all orders of $g$ have the property $(A C)$. As all the partial derivatives of order $h$ of $g$ are in $L_{\text {loc }}^{p}$ we can use the result by Deny-Lions [7, p. 315] and Kryloff's theorem as in $1^{\circ}$ to conclude that $g$ defines a distribution belonging to $B L_{n}\left(L_{\mathrm{loc}}^{p}\right)$. In particular we obtain that $g$ and all the partial derivatives of $g$ of orders less than $h$ are in $L_{\text {loc }}^{p}$ too.

$3^{\circ}$. As we in the extension problem only are interested in continuous functions we introduce two classes $\mathcal{A}_{h}\left(L_{\mathrm{loc}}^{p}\right)$ and $\mathcal{A}_{h}^{*}\left(L_{\mathrm{loc}}^{p}, F_{0}\right), p \geqslant 1$, of functions in the following ways: $A_{h}\left(L_{\mathrm{loc}}^{p}\right)$ is the class of functions $f$ defined and continuous everywhere whichconsidered as distributions - belong to $B L_{h}\left(L_{\mathrm{loc}}^{p}\right) . \mathcal{A}_{h}^{*}\left(L_{\mathrm{loc}}^{p}, F_{0}\right)$ is the class of functions $f$ defined and continuous everywhere in $R^{m}$, infinitely differentiable on $\mathbf{C} F_{0}$, and such that all the partial derivatives of $f$ of orders less than or equal to $h$ belong to $L_{10 \mathrm{lo}}^{p}$.

From the discussion in $2^{\circ}$ we conclude that $A_{h}^{*}\left(L_{\text {loc }}^{p}, F_{0}\right)$, with $C_{\alpha}\left(F_{0}\right)=0$ for some $\alpha<m-1$, is a subclass of $\mathcal{A}_{h}\left(L_{\mathrm{loc}}^{p}\right)$.

$4^{\circ}$. We shall need the following fact: Let $f_{0} \in S\left(F_{0}\right)$. If there exists a function $f \in A_{h}^{*}\left(L_{\text {loc }}^{p}, F_{0}\right)$ (or $\mathcal{A}_{h}\left(L_{\text {loc }}^{p}\right)$ ) coinciding pointwise with $f_{0}$ on $F_{0}$, then there exists a function $f^{*} \in \mathcal{A}_{h}^{*}\left(L_{\mathrm{loc}}^{p}, F_{0}\right)$ (or $\mathcal{A}_{h}\left(L_{\mathrm{loc}}^{p}\right)$ ) which is zero outside a compact set and coincides pointwise with $f_{0}$ on $F_{0}$.

In fact, as the function $f^{*}$ we only have to choose $f \varphi$, where $\varphi$ is identically 1 on $F_{0}$, infinitely differentiable and has a compact support.

\section{An extension theorem}

We need the following lemma:

Lemma 4. Let $0<\alpha<\beta<m$. Let $\mu$ be a positive measure with $\mu\left(R^{m}\right)<\infty$ and suppose that $u_{\alpha}^{\mu}$ is bounded. Then we have

$$
u_{\beta}^{\mu} \in L^{p} \text { if } 2 \leqslant p=\frac{m-\alpha}{\beta-\alpha}
$$

and

$$
u_{\beta}^{\mu} \in L_{100}^{p} \text { if } 1 \leqslant p<\frac{m-\alpha}{\beta-\alpha}<2 \text {. }
$$

More exactly, for $p>1$ we have, if $a=\mu\left(R^{m}\right)$,

$$
\left\|u_{\beta}^{\mu}\right\|_{L^{p}} \leqslant M_{1}(a) \cdot\left\{\sup _{x \in R^{m}} u_{\alpha}^{\mu}(x)\right\}^{(p-1) / p}, \text { if } 2 \leqslant p=\frac{m-\alpha}{\beta-\alpha}
$$

and for every sphere $S$ with radius $r$, we have

$$
\left\|u_{\beta}^{\mu}\right\|_{L^{p}(S)} \leqslant M_{2}(a, r) \cdot\left\{\sup _{x \in R^{m}} u_{\alpha}^{\mu}(x)\right\}^{(p-1) / p} \text {, if } 1<p<\frac{m-\alpha}{\beta-\alpha}<2 .
$$

$M_{1}(a)$ is a constant depending further on $m, p$ and $\alpha$ and $M_{2}(a, r) a$ constant depending further on $m, p, \alpha$ and $\beta$.

\footnotetext{
1 Compare for instance Frostman [9, p. 91] and Brelot [2, p. 330].
} 


\section{H. WALLIN, Continuous functions and potential theory}

For a proof of the lemma, based essentially on Hölders inequality, we refer to du Plessis [15], Deny [6] and Fuglede [10]. A proof based on function theory is given in Carleson $[4$, p. 61 and p. 80] for some special values of $\alpha$ and $\beta$. The method is, however, applicable in the general case, too.

Theorem 3. Let $F_{0}$ be a compact set, $p \geqslant 1$ and $h$ a positive integer. Suppose that either

$$
C_{\alpha}\left(F_{0}\right)=0 \text { for } \alpha=m-p h \text { where } m-p h \geqslant 0 \text { and } p \geqslant 2
$$

or

$C_{\alpha}\left(F_{0}\right)=0$ for some $\alpha$ satisfying $\alpha<m-p h$ where $m-p h>0$ and $1 \leqslant p<2$.

Then every function belonging to $\mathbf{S}\left(\boldsymbol{F}_{0}\right)$ can be extended to a function which is defined and continuous everywhere in $R^{m}$ and infinitely differentiable on $\mathbf{C} F_{0}$ and such that all the partial derivatives of orders less than or equal to $h$ of the extended function and the extended function itself are in $L^{p}$.

Proof. We first treat the case $\alpha>0$ and assume that either (6.5) or (6.6) is valid.

Suppose that $f_{0}$ is a function from $S\left(F_{0}\right)$. According to $\S 5,4^{\circ}$ it is enough to prove that there is a function from $\mathcal{A}_{h}^{*}\left(L_{\mathrm{loc}}^{p}, F_{0}\right)$ coinciding pointwise with $f_{0}$ on $F_{0}$.

As $C_{\alpha}\left(F_{0}\right)=0$ there exists an absolutely continuous measure $\sigma$, constructed as in Theorem 1, with compact support such that $u_{\alpha}^{\sigma}$ is continuous everywhere, infinitely differentiable on $\mathrm{C} F_{0}, u_{\alpha}^{|\sigma|}$ is bounded and $u_{\alpha}^{\sigma}(x)=f_{0}(x)$ for every $x \in F_{0}$. We shall prove that $u_{\alpha}^{\sigma} \in \mathcal{A}_{h}^{*}\left(L_{\text {loc }}^{p}, F_{0}\right)$. An easy consequence of the properties of $\sigma$ is that

$$
D^{s} u_{\alpha}^{\sigma}(x)=\int D_{x}^{s} \frac{1}{|x-y|^{\alpha}} d \sigma(y) \quad \text { for every } \quad x \in \mathbf{C} F_{0},
$$

for all sequences $s$ with $|s| \leqslant h$ if $\alpha+h<m$. The index $x$ in $D_{x}^{s}$ denotes derivation with respect to $x$.

(6.7) yields, if $|s|=h, \alpha+h<m$,

$$
\left|D^{s} u_{\alpha}^{\sigma}(x)\right| \leqslant M u_{\alpha+h}^{|\sigma|}(x) \quad \text { for every } \quad x \in \mathbf{C} F_{0},
$$

where $M$ is a constant only depending on $h, \alpha$ and $m$. As $u_{\alpha}^{|\sigma|}$ is bounded we can, by means of (6.8) and Lemma 4 used with $\beta=\alpha+h$, conclude that all the derivatives of order $h$ of $u_{\alpha}^{\sigma}$ belong to $L_{\text {loc }}^{p}$; we use $(6.1)$ if $(6.5)$ is valid and (6.2) if (6.6) is valid. From the discussion in $\S 5,2^{\circ}$ we finally conclude that $u_{\alpha}^{\sigma} \in \mathcal{A}_{h}^{*}\left(L_{\mathrm{loc}}^{p}, F_{0}\right)$, and so the theorem is proved if $\alpha>0$.

The case $\alpha=0$ is treated analogously to the case $\alpha>0$, by using the following lemma instead of Lemma 4:

Lemma 5. Let $0<\beta<m$. Let $\mu$ be a positive measure with compact support. If either $I_{0}(\mu)$ is finite and $2 \leqslant p=m / \beta$ or $1 \leqslant p<m / \beta$, then $u_{\beta}^{\mu} \in L_{\mathrm{loc}}^{p}$.

The proof of Lemma 5 is analogous to that of Lemma 4 (see Fuglede [10]).

\section{A converse of Theorem 3}

Theorem 3 gives a positive solution of our extension problem with the extended function in the class $\mathcal{A}_{h}^{*}\left(L_{\mathrm{loc}}^{p}, F_{0}\right)$ if $F_{0}$ satisfies (6.5) or (6.6). The following theorem gives a converse of Theorem 3 as $\mathcal{A}_{h}^{*}\left(L_{\mathrm{loc}}^{p}, F_{0}\right)$ is a subclass of $\mathcal{A}_{h}\left(L_{\mathrm{loc}}^{p}\right)$ if $C_{\alpha}\left(F_{0}\right)=0$ for some $\alpha<m-1$ : 
Theorem 4. Let $F_{0}$ be a compact set. A sufficient condition for the existence of a function in $S\left(F_{0}\right)$, which cannot be extended to a function in $\mathcal{A}_{h}\left(L_{\mathrm{loc}}^{p}\right)$, is that either

$$
C_{\alpha}\left(F_{0}\right)>0 \text { for some } \alpha \text { satisfying } \alpha>m-p h \text {, where } m-p h \geqslant 0 \text { and } p>2
$$

or

$$
C_{\alpha}\left(F_{0}\right)>0 \text { for } \alpha=m-p h \text { where } m-p h>0 \text { and } 1 \leqslant p \leqslant 2 \text {. }
$$

In order not to encumber the proof with details we shall not treat the case when $m-p h=0,1 \leqslant p \leqslant 2$. It is, however, no difficulty to modify the proof which we shall give to obtain: The theorem is also true in the case when

$$
C_{0}\left(F_{0}\right)>0, \quad m-p h=0, \quad 1 \leqslant p \leqslant 2 .
$$

An inspection of the Theorems 3 and 4 shows that they give a complete solution of our extension problem when $p=2$ but not when $p \neq 2$.

Theorem 4 is proved in $\S 8$. For the proof we need integral representations of those functions in $B L_{h}\left(L_{\mathrm{loc}}^{p}\right)$ which are zero outside a compact set. To deduce these we use the following formulas (Schwartz [17, part I, p. 47]): If $\Delta_{h}$ is the Laplace operator iterated $h$ times, $h \geqslant 1$, then we have, in the distribution sense, if $\delta$ is the Dirac measure and $M_{1}$ and $M_{2}$ are certain constants only depending on $h$ and $m$,

$$
\begin{gathered}
M_{1} \cdot \Delta_{h}|x|^{2 h-m}=\delta \text { if } m-2 h>0 \text { or } m-2 h<0, m \text { odd }, \\
M_{2} \cdot \Delta_{h}\left(|x|^{2 h-m} \log |x|\right)=\delta \text { if } m-2 h \leqslant 0, m \text { even } .
\end{gathered}
$$

We can write

$$
\Delta_{h}=\sum a_{s} D^{s} D^{s}
$$

where $a_{s}$ are constants and the sum is extended over a number of multiindices $s$ with $|s|=h$. If $f \in B L_{h}\left(L_{\text {loc }}^{p}\right)$ and $f$ has compact support we obtain, by means of (7.6), in the case when (7.4) is valid,

$$
f=\delta * f=M_{1} \Delta_{h}|x|^{2 h-m} * f=M_{1} \sum a_{s} D^{s} * D^{s}|x|^{2 h-m} * f=M_{1} \sum a_{s} D^{s}|x|^{2 h-m} * D^{s} f .
$$

The distributions $D^{s}|x|^{2 n-m}$ and $D^{s} f$ are functions and $D^{s} f$ has compact support. The convolution $D^{s}|x|^{2 h-m} * D^{s} f$ can consequently be written as an integral and as $D^{s} f \in L^{p}$, we get:

If $f \in B L_{h}\left(L_{\text {loc }}^{p}\right), f$ has compact support and the function $k_{s, h}$ is defined by $k_{s, h}(x)=$ $D^{s}|x|^{2 h-m}$, then there are functions $g_{s}$ with compact supports, $g_{s} \in L^{p}$, and constants $b_{s}$ so that

$$
f(x)=\sum b_{s} \int k_{s, h}(x-y) g_{s}(y) d y \text { a.e., if } m-2 h>0 \text { or } m-2 h<0, m \text { odd. }
$$

Analogously we get by (7.5)—with other values on the constants $b_{s}$-if the function $k_{s, h}^{*}$ is defined by $k_{s, h}^{*}(x)=D^{s}\left(|x|^{2 h-m} \log |x|\right)$,

$$
f(x)=\sum b_{s} \int k_{s, h}^{*}(x-y) g_{s}(y) d y \text { a.e., if } m-2 h \leqslant 0, m \text { even. }
$$

The sums in (7.7) and (7.8) are extended over a number of multiindices $s$ with $|s|=h$.

In the proof of Theorem 4 we also need the following lemma: 
H. WALLiN, Continuous functions and potential theory

Lemma 6. ${ }^{1}$ Let $K$ be a kernel. Suppose that $g_{i}, i=1,2$, are functions having the property that for every $\varepsilon>0$ there exists a Borel set $E$ with $C_{E}(E)<\varepsilon$ such that the restrictions of $g_{1}$ and $g_{2}$ to $\mathrm{C} E$ both are continuous. If we, furthermore, assume that $g_{1}(x)=g_{2}(x)$ a.e. and that the set of points $x$ where $g_{1}(x) \neq g_{2}(x)$ is a Borel set, then $g_{1}(x)=g_{2}(x)$ except on a set of $K$-capacity zero.

Proof. Let $E_{1}$ be the set of points $x$ where $g_{1}(x) \neq g_{2}(x)$ and suppose that $C_{K}\left(E_{1}\right)=$ $a>0$. Choose $\varepsilon, 0<\varepsilon<a$, and let $E$ be a Borel set with $C_{K}(E)<\varepsilon$ such that the restrictions of $g_{1}$ and $g_{2}$ to $C E$ both are continuous. We further choose a compact subset $F$ of $E_{1} \backslash E$ with $C_{R}(F)>a-\varepsilon$. Let $F_{n}$, for $n=1,2, \ldots, F_{n} \supset F$, be the union of finitely many closed spheres having radii $\leqslant r_{n}$, where $r_{n} \rightarrow 0$ when $n \rightarrow \infty$, and centres belonging to $F$ and let $F_{n}^{*}$ consist of the set of points situated at a distance $\leqslant r_{n}$ from $F_{n}$.

If $\mu_{n}$ is a capacitary distribution belonging to the kernel $K$ and $F_{n}$, then

$$
u_{K}^{\mu_{n}}(x) \leqslant A \cdot\left\{C_{K}\left(F_{n}\right)\right\}^{-1},
$$

where $A$ is the constant in (1.4). Let $\varphi_{n}, \int \varphi_{n} d x=1$, be a non-negative function which is infinitely differentiable and let $S_{\varphi_{n}}$ be a subset of $S\left(0, r_{n}\right)$. We define $\psi_{n}$ by $\psi_{n}=\varphi_{n} * \mu_{n} \cdot \psi_{n} d x$ is a positive measure with total mass 1 and $S_{\psi_{n}} \subset F_{n}^{*}$. Since $E_{1}$ has Lebesque measure zero there exists for every $\eta>0$ a closed set $H_{n}(\eta), H_{n}(\eta) \subset F_{n}^{*} \backslash E_{1}$, such that the restriction of the measure $\psi_{n} d x$ to $H_{n}(\eta)$ has total mass $\geqslant 1-\eta$. Hence

$$
\begin{gathered}
I_{K}\left(\psi_{n}\right) \geqslant(1-\eta)^{2}\left\{C_{K}\left(H_{n}(\eta)\right)\right\}^{-1} \geqslant(1-\eta)^{2}\left\{C_{K}\left(F_{n}^{*} \backslash E_{1}\right\}^{-1} \cdot \eta \rightarrow 0\right. \text { gives } \\
I_{K}\left(\psi_{n}\right) \geqslant\left\{C_{K}\left(F_{n}^{*} \backslash E_{1}\right)\right\}^{-1}
\end{gathered}
$$

On the other hand we have by (7.9)

$$
u_{K}^{\psi_{n}}(x)=K * \varphi_{n} * \mu_{n}(x)=\varphi_{n} * u_{K^{n}}^{\mu_{n}}(x) \leqslant A \cdot\left\{C_{K}\left(F_{n}\right)\right\}^{-1},
$$

which gives $I_{K}\left(\psi_{n}\right) \leqslant A \cdot\left\{C_{K}\left(F_{n}\right)\right\}^{-1}$. This inequality and (7.10) give

$$
C_{K}\left(F_{n}^{*} \backslash E_{1}\right) \geqslant A^{-1} C_{K}\left(F_{n}\right) \geqslant A^{-1} C_{K}\left(F^{\prime}\right)>A^{-1} \cdot(a-\varepsilon) .
$$

By choosing $\varepsilon$ so small that $A^{-1}(a-\varepsilon)>\varepsilon$ we get $C_{K}\left(F_{n}^{*} \backslash E_{1}\right)>C_{K}(E)$ as $C_{K}(E)<\varepsilon$, i.e. the set $\left(F_{n}^{*} \backslash E_{1}\right) \backslash E$ is non-empty.

Let $x_{n} \in\left(F_{n}^{*} \backslash E_{1}\right) \backslash E$. According to our construction we have

$$
g_{1}\left(x_{n}\right)=g_{2}\left(x_{n}\right), \quad n=1,2, \ldots, \text { and }
$$

There exists a point $y_{n} \in F$ such that $\left|x_{n}-y_{n}\right| \leqslant 2 r_{n}, \quad n=1,2, \ldots$

We now prove the existence of a point $x_{0}$ from $F$ where $g_{1}$ and $g_{2}$ coincide which gives a contradiction to the fact that $F \subset E_{1}$. We suppose that the sequence of points $\left\{x_{n}\right\}$ converges to a point $x_{0}$. (If this is not the case, we choose a convergent subsequence.) From (7.12) we conclude that $\left\{y_{n}\right\}$ converges to $x_{0}$ too. Hence $x_{0} \in F$. We now use the estimate

$$
\left|g_{1}\left(x_{0}\right)-g_{2}\left(x_{0}\right)\right| \leqslant\left|g_{1}\left(x_{0}\right)-g_{1}\left(x_{n}\right)\right|+\left|g_{1}\left(x_{n}\right)-g_{2}\left(x_{n}\right)\right|+\left|g_{2}\left(x_{n}\right)-g_{2}\left(x_{0}\right)\right| \text {. }
$$

1 A special case of this lemma is given in Deny and Lions [7, p. 353]. 
The second term of the right member is zero according to (7.11). As $x_{n}$ and $x_{0}$ belong to $\mathrm{C} E$ and the restrictions of $g_{1}$ and $g_{2}$ to $\mathrm{C} E$ are continuous, we conclude that the two remaining terms of the right member are arbitrarily small when $n$ is large. This proves that $g_{1}\left(x_{0}\right)=g_{2}\left(x_{0}\right)$ which gives a contradiction to the fact that $x_{0} \in F$.

\section{Proof of Theorem 4 and a theorem on harmonic functions}

The idea of the proof of Theorem 4 is as follows: We start by observing that, due to $\S 5,4^{\circ}$, we only have to prove the existence of a function $f_{0} \in S\left(F_{0}\right)$ which cannot be extended to a function in $\mathcal{A}_{h}\left(L_{\mathrm{loc}}^{p}\right)$ with compact support if (7.1) or (7.2) is satisfied. But every function in $\mathcal{A}_{h}\left(L_{\mathrm{loc}}^{v}\right)$ is-considered as a distribution-an element in $B L_{h}\left(L_{\mathrm{loc}}^{p}\right)$. This means that every function in $\mathcal{A}_{h}\left(L_{\mathrm{loc}}^{p}\right)$ with compact support can be written on the form (7.7) or (7.8). Following the method of the proof of Theorem 2 we shall deduce moduli of continuity of the right members of (7.7) and (7.8) having properties analogous to those of the modulus of continuity of the potentials in the proof of Theorem 2. This will also show that the right members of (7.7) and (7.8) have such properties that we can use Lemma 6 to conclude that every function in $A_{h}\left(L_{\mathrm{loc}}^{p}\right)$ with compact support has a representation (7.7) or (7.8) valid not only a.e. but everywhere except on a set of $K_{0}$-capacity zero, where the kernel $K_{0}$ is defined below. (Compare (8.1) and (8.17).) The choice of $K_{0}$ is such that $C_{K_{0}}\left(F_{0}\right)>0$ and this will enable us to infer, from Lemma 3 , the existence of a function $f_{0} \in S\left(F_{0}\right)$ which cannot be extended to a function in $A_{h}\left(L_{\mathrm{loc}}^{p}\right)$ if (7.1) or (7.2) is valid.

The case $1 \leqslant p \leqslant 2$. We start by treating the case $1 \leqslant p \leqslant 2$ and we suppose consequently that (7.2) is true for the given set $F_{0}$ and given values on $h$ and $p, 1 \leqslant p \leqslant 2$. Since $C_{\alpha}\left(F_{0}\right)>0$ for $\alpha=m-p h$ there exists a kernel $K_{0}$ satisfying

$$
\lim _{r \rightarrow 0} K_{0}(r) r^{m-p h}=\infty, C_{K_{0}}\left(F_{0}\right)>0, \lim _{r \rightarrow \infty} K_{0}(r)>0 .
$$

As $C_{K_{0}}\left(F_{0}\right)>0$ we can choose a measure $\nu_{0}$ with

$$
\nu_{0} \geqslant 0, \quad \nu_{0}\left(R^{m}\right)=1, \quad S_{\nu_{0}} \subset F_{0}, \quad I_{K_{0}}\left(\nu_{0}\right)<\infty .
$$

We formulate the information which we need about the right members of (7.7) and (7.8) in the following lemma:

Lemma 7. Let the compact set $F_{0}$, the kernel $K_{0}$, and the measure $v_{0}$ satisfy (8.1) and (8.2) and suppose that $p$ and $h$ are given numbers, $h$ a positive integer, with $m-p h>0$, $1 \leqslant p \leqslant 2$. Using the notations of (7.7) and (7.8) we define the function $v_{h}$ by

$$
v_{h}(x)=\sum b_{s} \int k_{s, h}(x-y) g_{s}(y) d y \quad \text { if } \quad m-2 h>0 \quad \text { or } \quad m-2 h<0, m \quad \text { odd, }
$$

and by

$$
v_{h}(x)=\sum b_{s} \int k_{s, h}^{*}(x-y) g_{s}(y) d y \text { if } m-2 h \leqslant 0, m \text { even, }
$$

at those points where the right members are well defined. The sums are extended over the same multiindices $s$ as in (7.7) and (7.8), i.e. over a number of $s$ with $|s|=h ; b_{s}$ are constants and $g_{s}$ functions in $L^{p}$ with compact supports. Then there exists a non-negative function $t_{h}$ defined in the interval $r>0, \lim _{r \rightarrow 0} t_{h}(r)=0$, only depending on $m, h$ and $K_{0}$ such that the following assertion is true: 
H. WALLIN, Continuous functions and potential theory

For every $\varepsilon>0$ there exists a Borel set $E_{1}$ with $C_{K_{0}}\left(E_{1}\right)<\varepsilon$ and a constant $M$ only depending on $\varepsilon, m, h, K_{0}, b_{s}$ and $g_{s}$ so that

$$
\left|v_{h}\left(x_{1}\right)-v_{h}\left(x_{2}\right)\right| \leqslant M t_{h}\left(\left|x_{1}-x_{2}\right|\right) \text { for all } x_{1}, x_{2} \in \mathcal{C} E_{1} .
$$

Furthermore, there exists a.Borel set $E_{2}$ with $\nu_{0}\left(E_{2}\right)<\varepsilon$ such that (8.5) is valid with $E_{1}$ replaced by $E_{2}$.

We can easily finish the proof of Theorem 4 in the case $1 \leqslant p \leqslant 2$ if for a moment we suppose that Lemma 7 has been proved. As we have mentioned above it is enough to prove the existence of a function $f_{0} \in S\left(F_{0}\right)$ which cannot be extended to a function in $\mathcal{A}_{h}\left(L_{\mathrm{loc}}^{p}\right)$ with compact support. To prove this we first study those functions in $\mathcal{A}_{h}\left(L_{\text {loc }}^{p}\right)$ which have compact supports. Let $f$ be such a function. Then $f$ has a representation (7.7) if $m-2 h>0$ or $m-2 h<0, m$ odd, and a representation (7.8) if $m-2 h \leqslant 0$, $m$ even. This gives an integral representation of $f$ valid a.e. But since $f$ is continuous we can use (8.5) of Lemma 7 and Lemma 6 to conclude that this integral representation of $f$ is valid everywhere except on a set of $K_{0}$-capacity zero. ${ }^{1}$ By means of the last sentence of Lemma 7 we infer, as the set where the integral representation of $f$ is not true has $K_{0}$-capacity zero and accordingly also $v_{0}$-measure zero: For every $\varepsilon>0$ there exists a Borel set $E, E \subset F_{0}, v_{0}(E)<\varepsilon$, such that, if $t_{h}$ is the function occurring in Lemma 7 , then

$$
\left|f\left(x_{1}\right)-f\left(x_{2}\right)\right| \leqslant M t_{h}\left(\left|x_{1}-x_{2}\right|\right) \text { for all } x_{1}, x_{2} \in F_{0}-E .
$$

Using Lemma 3 and (8.6) the proof is now completed in the same way as we finished the proof of Theorem 2 by means of Lemma 3.

It remains to prove Lemma 7.

Proof of Lemma 7 . We first deal with the case $p=2$. According to our assumptions we have $m-2 h>0$ which means that the case when $v_{h}$ is defined by (8.4) does not occur. The kernel $K_{0}$ has when $p=2$ the properties

$$
\lim _{\tau \rightarrow 0} K_{0}(r) r^{m-2 h}=\infty, C_{K_{0}}\left(F_{0}\right)>0, \lim _{r \rightarrow \infty} K_{0}(r)>0 .
$$

Let $q$ be a function defined in the interval $r>0$ satisfying

$q$ is non-increasing, non-negative and continuous, $q(r) \leqslant A_{1} q(2 r)$ for every $r>0$,

$$
A_{1} \text { constant, } \lim _{r \rightarrow 0} q(r)=\infty .
$$

We define the function $g$ by

$$
g(y)=\sum\left|g_{s}(y)\right|
$$

where the sum is extended over those functions $g_{s}$ which occur in (8.3). Finally we introduce the function $w_{h}$ :

$$
w_{h}(x)=\int \frac{q(|x-y|) g(y)}{|x-y|^{m-h}} d y .
$$

\footnotetext{
1 Due to the degree of arbitrariness in the choice of $K_{0}$ in (8.1) it is, in fact, easy to realize, that the integral representation of $f$ holds true except on a set of $\alpha$-capacity zero with $\alpha=m-p h$.
} 
The reason for introducing the function $w_{h}$ is the following: Since $k_{s, h}(x)=D^{s}|x|^{2 h-m}$, $|s|=h$, we have

$$
\left|k_{s, h}(x)\right| \leqslant M \cdot|x|^{n-m}{ }^{1}
$$

This and (8.3) show that $v_{n}$ is majorized by a potential generated by the kernel $r^{h-m}$. But $w_{h}$ is a potential generated by the kernel $r^{h-m} \cdot q(r)$. Since $q(r)$ tends to infinity as $r$ tends to zero we may proceed as in the proof of Lemma 2 to show an inequality of the type of (8.5) for $v_{h}$, valid for points $x_{1}$ and $x_{2}$ from a set of points where $w_{n}$ is majorized by a certain constant. We start by performing this and then we shall finish the proof of Lemma 7 in the case $p=2$ by showing that $w_{h}$ is bounded except on a set having $K_{0}$-capacity and $v_{0}$-measure less than a prescribed positive number, if $q$ is properly chosen.

We accordingly suppose that $w_{h}\left(x_{i}\right) \leqslant a, i=1,2$, where $a$ is a given positive number. As in the proof of Lemma 2 we put $\left|x_{1}-x_{2}\right|=r_{0}$ and introduce the open sphere $S$ with centre $2^{-1}\left(x_{1}+x_{2}\right)$ and radius $2^{-1} r_{0}+r_{1}, r_{1}>r_{0}$, and write

$$
v_{h}\left(x_{1}\right)-v_{h}\left(x_{2}\right)=\int_{S}+\int_{G S}=I+I I .
$$

Using (8.9) we obtain

$$
|I| \leqslant a \cdot M\left\{q\left(r_{0}+r_{1}\right)\right\}^{-1} .
$$

If $y \in C S$ we obtain, by using the mean value theorem, if $|s|=h$,

$$
\left|k_{s, h}\left(x_{1}-y\right)-k_{s, h}\left(x_{2}-y\right)\right| \leqslant M \frac{\left|x_{1}-x_{2}\right|}{\left(r_{1}-r_{0}\right)^{m-n+1}} .
$$

As in the proof of Lemma 2 we now realize that we can choose $r_{1}$ depending on $r_{0}$ and find a function $t_{h}$, defined in the interval $r>0, t_{h}(r) \rightarrow 0$ when $r \rightarrow 0$, only depending on $m, h$ and $q$, such that

$$
\left|v_{h}\left(x_{1}\right)-v_{h}\left(x_{2}\right)\right| \leqslant(a+1) M t_{h}\left(\left|x_{1}-x_{2}\right|\right) \quad \text { if } \quad w_{h}\left(x_{i}\right) \leqslant a, i=1,2 .
$$

The lemma follows, in the case $p=2$, by means of (8.10), if we prove that it is possible to choose $q$ only depending on $K_{0}$ such that, for every $\varepsilon>0$, there exists a constant $a$ with $w_{h}(x) \leqslant a$ except on a set of $K_{0}$-capacity and $\nu_{0}$-measure less than $\varepsilon$. But this is a consequence of the assertion that we can choose $q$ only depending of $K_{0}$ such that, if $G_{a}$ denotes the set where $w_{h}(x)>a>0$, then

$$
C_{K_{0}}\left(G_{a}\right)<M \cdot a^{-2}\|g\|_{L^{2}}^{2}
$$

To prove this last assertion we consider an arbitrary positive measure $\mu$ with $\mu\left(R^{m}\right)=1, S_{\mu} \subset G_{a}$. We have, by Schwarz's inequality,

$$
\begin{aligned}
a^{2} & <\left(\int w_{h}(x) d \mu(x)\right)^{2}=\left\{\int g(y)\left(\int \frac{q(|x-y|)}{|x-y|^{m-h}} d \mu(x)\right) d y\right\}^{2} \\
& \leqslant\|g\|_{L^{2}}^{2} \cdot \iint d \mu(x) d \mu(z) \int \frac{q(|x-y|) q(|z-y|)}{|x-y|^{m-h}|z-y|^{m-h}} d y .
\end{aligned}
$$

1 In the proof of this lemma $M$ denotes a constant-not necessarily the same each time it occurs-only depending on the parameters shown for the constant $M$ in Lemma 7. 
H. WALLIN, Continuous functions and potential theory

We define $H$ by $\quad H(x, z)=\int \frac{q(|x-y|) q(|z-y|)}{|x-y|^{m-h}|z-y|^{m-h}} d y$

and shall prove that we can choose $q$, satisfying (8.8) and only depending on $K_{0}$ such that

$$
H(x, z) \leqslant M K_{0}(|x-z|) .
$$

In fact, if for a moment we suppose that this has been proved we obtain from our estimate of $a^{2}$,

$$
a^{2}<\|g\|_{L^{2}}^{2} \cdot M \cdot I_{K_{0}}(\mu) .
$$

Hence

$$
a^{2}<M \cdot\|g\|_{L^{2}}^{2} \cdot\left\{C_{K_{0}}\left(G_{a}\right)\right\}^{-1}
$$

which yields (8.11).

We now prove the assertion leading to (8.12). If we put $z-x=\xi$ we obtain, by a substitution in the integral defining $H(x, z)$,

$$
H(x, z)=\int \frac{q(|y|) q(|y-\xi|)}{|y|^{m-h}|y-\xi|^{m-h}} d y .
$$

We denote this expression by $H_{1}(\xi)$ and estimate $H_{1}(\xi)$ by the following division of the integral, where $D$ is the set of points $y$ satisfying

$$
\begin{gathered}
|y|>\frac{|\xi|}{2},|y-\xi|>\frac{|\xi|}{2} \text { and }|y|<2|\xi|, \\
H_{1}(\xi)=\int_{|y| \leqslant \frac{|\xi|}{2}}+\int_{|y-\xi| \leqslant \frac{|\xi|}{2}}+\int_{|y| \geqslant 2|\xi|}+\int_{D}=I+I I+I I I+I V .
\end{gathered}
$$

By using $q(r) \leqslant A_{1} q(2 r)$ we obtain

$$
\begin{aligned}
I & \leqslant 2^{m-h} A_{1} q(|\xi|)|\xi|^{h-m} \int_{|y| \leqslant \frac{|\xi|}{2}}|y|^{h-m} q(|y|) d y \\
& \leqslant M A_{1} q(|\xi|)|\xi|^{h-m} \int_{0}^{|\xi|} r^{h-1} q(r) d r .
\end{aligned}
$$

For $I I$ we obtain the same estimate as for $I$.

$$
I I I \leqslant 2^{m-h}\{q(|\xi|)\}^{2} \cdot \int_{|y| \geqslant 2|\xi|} \frac{d y}{|y|^{2 m-2 h}} \leqslant M|\xi|^{2 h-m}\{q(|\xi|)\}^{2},
$$

as $m-2 h>0$.

$$
I V \leqslant A_{1}^{2} \cdot 2^{2 m-2 h}\{q(|\xi|)\}^{2} \cdot|\xi|^{2 h-2 m} \int_{D} d y \leqslant M \cdot A_{1}^{2} \cdot|\xi|^{2 h-m}\{q(|\xi|)\}^{2}
$$

These estimates show that $(\mathbf{8 . 1 2})$ is true if we can choose $q$ satisfying (8.8) so that

$$
q(|\xi|) \cdot|\xi|^{-n} \int_{0}^{|\xi|} r^{h-1} q(r) d r+\{q(|\xi|)\}^{2} \leqslant M K_{0}(|\xi|) \cdot|\xi|^{m-2 h},|\xi| \neq 0 .
$$


But this is a consequence of the facts that $m-2 h>0, \lim _{r \rightarrow 0} K_{0}(r) r^{m-2 h}=\infty$, $\lim _{r \rightarrow \infty} K_{0}(r)>0$ and, for instance, the fact that

$$
|\xi|^{-n} \int_{0}^{|\xi|} r^{h-1} q(r) d r \leqslant M_{2} q(|\xi|),
$$

if $q$ satisfies (8.8) with $A_{1}$ sufficiently close to $1 . M_{2}$ is a constant depending on $A_{1}$ and $h$. (8.13) is proved by dividing the integration interval $(0,|\xi|)$ by the points $2^{-n}|\xi|, n=0,1,2, \ldots$

Hence Lemma 7 is proved in the case $p=2$.

We now prove Lemma 7 when $1 \leqslant p<2$. In this case it may occur that $m-2 h \leqslant 0$. Consequently we also have to consider the case when the function $v_{h}$ is defined by the formula (8.4).

We let $q$ be a function satisfying (8.8) and introduce the functions $g$ and $w_{h}$ in the same way as in the case $p=2$. (8.10) is, of course, still valid, when $m-2 h>0$ or $m-2 h<0, m$ odd, i.e. when $v_{h}$ is defined by the formula (8.3), but we can also deduce the same formula when $m-2 h \leqslant 0, m$ even, i.e. when $v_{h}$ is defined by the formula (8.4). In fact, when $m-2 h \leqslant 0, m$ even, and $m-h>0$ it is easy to prove that the estimate (8.9) holds also for $k_{s, h}^{*}$ i.e. that

$$
\left|k_{s, h}^{*}(x)\right| \leqslant M|x|^{h-m},|s|=h .
$$

Analogously to the proof of (8.10) when $v_{h}$ is defined by (8.3) it is possible to show, by means of (8.14), the existence of a function $t_{h}$, defined in the interval $r>0$, $\lim _{r \rightarrow 0} t_{h}(r)=0$, only depending on $m, h$ and $q$ such that (8.10) is true also when $v_{h}$ is defined by (8.4).

An inspection of the proof when $p=2$ now shows that the lemma follows in the case $1 \leqslant p<2$ if we prove that we can choose $q$ only depending on $K_{0}$ such that the following substitute of (8.11) is valid,

$$
C_{K_{0}}\left(G_{a}\right)<M_{3} a^{-p}\|g\|_{L}^{p}, a>0,
$$

where $G_{a}$ denotes the set of points $x$ where $w_{h}(x)>a$ and $M_{3}$ is a constant depending on the same parameters as the constant $M$ in (8.5) and on the constant $A$ in (1.4).

To prove (8.15) we consider, as in the proof of (8.11), a positive measure $\mu, \mu\left(R^{m}\right)=1$, $S_{\mu} \subset G_{a}$. By using Hölder's inequality twice we have in the case $1<p<2,1$ if $p^{\prime}=p(p-1)^{-1}$ :

$$
\begin{aligned}
a^{p^{\prime}} & <\left(\int w_{h}(x) d \mu(x)\right)^{p^{\prime}} \leqslant\|g\|_{L^{\prime}}^{p^{\prime}} \int\left\{\int \frac{q(|x-y|)}{|x-y|^{m-h}} d \mu(x)\right\}^{p^{\prime}} d y \\
& =\|g\|_{L^{\prime} p}^{p^{\prime}} \int\left\{\int|x-y|^{\frac{(p h-m)\left(p^{\prime}-2\right)}{p^{\prime}}} \cdot|x-y|^{\frac{p h-2 m}{p^{\prime}}} \cdot q(|x-y|) d \mu(x)\right\}^{p^{\prime}} d y \\
& \leqslant\|g\|_{L^{\prime}}^{p^{\prime}} \int\left\{\int|x-y|^{p h-m} d \mu(x)\right\}^{p^{\prime}-2} \cdot\left\{\int|x-y|^{\frac{p h}{2}-m} q(|x-y|)^{\frac{p^{\prime}}{2}} d \mu(x)\right\}^{2} d y \\
& \leqslant\|g\|_{L^{\prime}}^{p^{\prime}}\left\{\sup _{y \in R^{m}} u_{m-p h}^{\mu}(y)\right\}^{p^{\prime}-2} \int\left\{\int|x-y|^{\frac{p h}{2}-m} \cdot q(|x-y|)^{\frac{p^{\prime}}{2}} d \mu(x)\right\}^{2} d y .
\end{aligned}
$$

\footnotetext{
1 As to the latter use of Hölder's inequality, compare [15, p. 130]. The case $p=1$ requires a simple special treatment which we omit.
} 
Defining $q_{1}$ by $q_{1}(r)=\{q(r)\}^{\frac{p^{*}}{2}}$ we get

$$
\begin{aligned}
& \int\left\{\int|x-y|^{\frac{p h}{2}-m} \cdot q\left(\left.|x-y|\right|^{\frac{p^{\prime}}{2}} d \mu(x)\right\}^{2} d y\right. \\
= & \iint d \mu(x) d \mu(z) \int \frac{q_{1}(|x-y|) \cdot q_{1}(|z-y|)}{|x-y|^{m-\frac{p h}{2}}|z-y|^{m-\frac{p h}{2}}} d y .
\end{aligned}
$$

If we replace $h$ by $p h / 2$ in the proof of (8.12) we realize that we can choose $q$ satisfying (8.8) such that the last integral above is majorized by $M \cdot I_{K_{0}}(\mu)$ where $K_{0}$ satisfies (8.1). Hence

$$
a^{p^{\prime}}<M\|g\|_{L p}^{p^{\prime}} \cdot \sup _{y \in \mathbb{R}^{m}}\left\{u_{m-p h}^{\mu}(y)\right\}^{p^{\prime}-2} \cdot I_{K_{0}}(\mu)
$$

Now let $F$ be an arbitrary closed subset of $G_{a}$. For $\mu$ we choose a capacitary distribution belonging to the kernel $K_{0}$ and the set $F$. This gives

$$
\sup _{y \in R^{m}} u_{m-p h}^{\mu}(y) \leqslant M \sup _{y \in R^{m}} u_{K_{0}}^{\mu}(y) \leqslant M \cdot A \cdot\left\{C_{K_{0}}(F)\right\}^{-1},
$$

where $A$ is the constant in (1.4). Consequently (8.16) yields

$$
a^{p^{\prime}}<M A^{p^{\prime}-2}\|g\|_{L^{p}}^{p^{\prime}}\left\{C_{K_{0}}(F)\right\}^{-p^{\prime}+1},
$$

i.e.

$$
C_{K_{v}}(F)<M_{4} a^{-p}\|g\|_{L p}^{p} .
$$

Since $F$ is an arbitrary closed subset of $G_{a}$ we conclude that (8.15) holds true.

By that Lemma 7 is proved and accordingly also Theorem 4 in the case $1 \leqslant p \leqslant 2$.

The case $p>2$. We now prove Theorem 4 in the case $p>2$ and we consequently assume that (7.1) is valid. According to (7.1) we can choose $\varepsilon_{0}$ such that

$$
C_{\alpha_{0}}\left(F_{0}\right)>0, \alpha_{0}=m-p h+\varepsilon_{0}, \alpha_{0}<m, \varepsilon_{0}>0 .
$$

Furthermore, we choose a positive measure $v_{0}$ having the properties

$$
\nu_{0} \geqslant 0, \nu_{0}\left(R^{m}\right)=1, S_{v_{0}} \subset F_{0}, I_{\alpha_{0}}\left(\nu_{0}\right)<\infty .
$$

The proof is now analogous to the proof of the theorem in the case $1 \leqslant p \leqslant 2$ if we replace the kernel $K_{0}$ by $r^{-\alpha_{0}}$ and use the following lemma instead of Lemma 7.1

Lemma 8. Let the compact set $F_{0}$, the number $\alpha_{0}$ and the measure $\nu_{0}$ satisfy (8.17) and (8.18). Suppose that $p$ and $h, h$ a positive integer, are given numbers with $m-p h \geqslant 0, p>2$. If we define the function $v_{h}$ by the same formula (8.3) as in Lemma 7 , then exactly the same conclusions as in Lemma 7 are true, word by word, concerning $v_{h}$ if we replace the kernel $K_{0}$ occurring in Lemma 7 by $r^{-\alpha_{0}}$.

\footnotetext{
1 Observe that the case when the function $v_{h}$ is defined by (8.4) does not occur when $p>2$ since $m-2 h>0$ if $p>2$, due to our assumption (7.1).
} 
The proof of Lemma 8 is analogous to the proof of Lemma 7 for the case $p=2$. The differences are that the function $w_{h}$ which is used in the proof of Lemma 7 now is defined by

$$
w_{h}(x)=\int \frac{g(y)}{|x-y|^{\gamma}} d y, \gamma=m-h+\varepsilon_{0}^{\prime}, \varepsilon_{0}^{\prime}>0,
$$

where $g$ is defined in the same way as in the proof of Lemma 7, and that we, instead of (8.11), use the fact that if $\varepsilon_{0}^{\prime}$ is chosen small enough and $G_{a}$ as usual denotes the set of points $x$ where $w_{h}(x)>a>0$, then

$$
C_{\alpha_{a}}\left(G_{a}\right)<M a^{-p}\|g\|_{L^{p}}^{p}
$$

This fact is proved by using formula (6.4) in Lemma 4 and as the result can be extracted from du Plessis $[15, \S 5]$ and Fuglede $[10, \S 4]$ we omit the details.

By that Theorem 4 is completely proved.

We now use Theorem 4 to prove the following theorem on harmonic functions.

Theorem 5. Let $U$ be the open unit sphere in $R^{m}, m \geqslant 2$, and $F_{0}$ a closed subset of the boundary of $U$. Then $C_{m-2}\left(F_{0}\right)=0$ if and only if every function $f_{0} \in S\left(F_{0}\right)$ can be extended to a function $u_{0}$ which is harmonic in $U$, continuous in the closure of $U$, has a finite Dirichlet integral

$$
\int_{v i=1}^{m}\left(\frac{\partial u_{0}}{\partial x^{i}}\right)^{2} d x<\infty
$$

and satisfies $u_{0}(x)=f_{0}(x)$ for every $x \in F_{0}$.

Proof. One half of the theorem has been proved in $\$ 2$, Remark 4. To prove the other half we assume that $C_{m-2}\left(F_{0}\right)>0$ and assert that there exists a function $f_{0} \in S\left(F_{0}\right)$ which cannot be extended to a function $u_{0}$ having the properties stated in the theorem.

Let $u$ be a function which is continuous in the closure of $U$, harmonic in $U$ and such that (8.19) holds with $u_{0}$ replaced by $u$. We define the function $u^{*}$ by

$$
u^{*}(x)=\left\{\begin{array}{l}
u(x) \text { if }|x| \leqslant 1, \\
u\left(\frac{x}{|x|^{2}}\right) \text { if }|x|>1, \text { where } \frac{x}{|x|^{2}}=\left(\frac{x^{1}}{|x|^{2}}, \ldots, \frac{x^{m}}{|x|^{2}}\right) .
\end{array}\right.
$$

Our assertion clearly follows from Theorem 4 if we prove that $u^{*} \in \mathcal{A}_{1}\left(L_{\text {loc }}^{2}\right)$. We first observe that all the partial derivatives of the first order of $u^{*}$ are in $L_{\text {loc }}^{2}$. Furthermore, it is easy to realize that $u^{*}$ has the property $(A C)$. In fact, if $a$ and $b$ are given finite numbers, we have on almost every line with the direction $x^{i}$

$$
\int_{a}^{b}\left(\frac{\partial u^{*}}{\partial x^{i}}\right)^{2} d x^{i}<\infty
$$

which proves that $u^{*}$ is absolutely continuous on almost every line with the direction $x^{i}$. 
Since $u^{*}$ has the property $(A C)$ and all the partial derivatives of the first order of $u^{*}$ are in $L_{\text {loc }}^{2}$, we conclude (compare Deny-Lions [7, p. 315]) that $u^{*} \in \mathcal{A}_{1}\left(L_{\mathrm{loc}}^{2}\right)$, which proves our theorem.

\section{The case $p=1$}

Theorem 3 gives, for $p=1$, a result in one direction of our investigation of the extension problem in the case when $C_{\alpha}\left(F_{0}\right)=0$ for some $\alpha<m-h$, and Theorem 4 a result in the other direction when $C_{\alpha}(F)>0$ for $\alpha=m-h$. We now give some additional results when $C_{\alpha}\left(F_{0}\right)=0$ for $\alpha=m-h$ but $C_{\alpha}\left(F_{0}\right)>0$ for every $x<m-h$.

We start by the following example'

Example 1. Let $F_{0}$ be the set of points $x=\left(x^{1}, \ldots, x^{m}\right)$ with $x^{1}=2^{-n}$ for $n=0,1,2, \ldots$ or $x^{1}=0$ and $0 \leqslant x^{i} \leqslant 1, i=2, \ldots, m$. We choose a sequence $\left\{a_{i}\right\}_{0}^{\infty}$ such that $\sum_{0}^{\infty} a_{i}$ is convergent and $\sum_{0}^{\infty}\left|a_{i}\right|=\infty$ and define a function $f_{0} \in \mathcal{S}\left(F_{0}\right)$ by putting, for $n=0,1, \ldots$,

$$
\begin{aligned}
& f_{0}(x)=\sum_{0}^{n} a_{i} \text { if } x \in F_{0}, x^{1}=2^{-n} \\
& f_{0}(x)=\sum_{0}^{\infty} a_{i} \text { if } x \in F_{0}, x^{1}=0 .
\end{aligned}
$$

and

If $f$ is an extension of $f_{0}$ to $R^{m}$ which is continuous everywhere and infinitely differentiable on $\mathbf{C} F_{0}$, we obtain, for $0 \leqslant x^{i} \leqslant 1, i=2, \ldots, m$,

$$
\int_{0}^{1}\left|\frac{\partial f\left(x^{1}, \ldots, x^{m}\right)}{\partial x^{1}}\right| d x^{1}=\sum_{0}^{\infty} \int_{2^{-n-1}}^{2^{-n}}\left|\frac{\partial f\left(x^{1}, \ldots, x^{m}\right)}{\partial x^{1}}\right| d x^{1} \geqslant \sum_{0}^{\infty}\left|a_{n+1}\right|
$$

and hence

$$
\int_{0}^{1} \ldots \int_{0}^{1}\left|\frac{\partial f\left(x^{1}, \ldots, x^{m}\right)}{\partial x^{1}}\right| d x^{1} \ldots d x^{m}=\infty \text {. }
$$

The given set $F_{0}$ is thus an example of a compact set which has $\alpha$-capacity zero for $\alpha=m-1$, if $m \geqslant 2$, is enumerable if $m=1$, and has the following property: There exists a function $f_{0} \in S\left(F_{0}\right)$ that cannot be extended to a function which is continuous everywhere, infinitely differentiable on $\mathbf{C} F_{0}$ and such that all the partial derivatives of the first order of the extended function are in $L_{\mathrm{loc}}^{1}$.

We use the above example to prove the following theorem:

Theorem 6. Let $h$ be a positive integer, $\alpha=m-h$ and $\alpha \geqslant 0$. Then there exists a compact set $F_{0}$ which satisfies $C_{\alpha}\left(F_{0}\right)=0$ if $\alpha>0$ and is enumerable if $\alpha=0$, and a function $f_{0} \in S\left(F_{0}\right)$ that cannot be extended to a function $f$ which is continuous everywhere in $R^{m}$, infinitely differentiable on $\mathbf{C} F_{0}$ and such that all the partial derivatives of order $h$ of $f$ belong to $L_{\mathrm{loc}}^{1}$.

Proof. For $h=1$ the theorem is a consequence of Example 1. Hence we assume that $h>1$.

\footnotetext{
1 The idea to use an example of this kind has been proposed to me by Dr. G. Aronsson.
} 
Put $m^{*}=m-(h-1)$ and let $\mathbf{R}^{m *}$ be the $m^{*}$-dimensional subspace of $R^{m}$ which is defined by the points $\left(x^{1}, \ldots, x^{m *}, 0, \ldots, 0\right)$ where $x^{1}, \ldots, x^{m^{*}}$ varies arbitrarily. According to Example 1 we can then choose a compact set $F_{0}, F_{0} \subset R^{m *}$, which satisfies $C_{\alpha}\left(F_{0}\right)=0$ if $\alpha>0$ and is enumerable if $\alpha=0$, and a function $f_{0} \in S\left(F_{0}\right)$ which cannot be extended to a function $g$, which is defined and continuous in $R^{m^{*}}$, infinitely differentiable on $R^{m^{*}}-F_{0}$ and such that

$$
\int_{a}^{b} \ldots \int_{a}^{b}\left|\frac{\partial g\left(x^{1}, \ldots, x^{m *}\right)}{\partial x^{1}}\right| d x^{1} \ldots d x^{m *}<\infty
$$

for all finite values of $a$ and $b$.

Suppose that we have chosen $F_{0}$ and $f_{0}$ as indicated and that there exists an extension $f$ of $f_{0}$ having the properties stated for the function $f$ in the theorem. We shall prove that this gives a contradiction by studying the derivative

$$
\frac{\partial^{h} f}{\partial x^{1} \partial x^{m^{*}+1} \partial x^{m^{\star}+2} \ldots \partial x^{m}}
$$

For every choice of $x^{1}, \ldots, x^{m-1}$ such that the line through the point $\left(x^{1}, \ldots, x^{m-1}, 0\right)$ parallel to the $x^{m}$-axis does not intersect $F_{0}$, we get,

$$
\int_{0}^{t_{0}} \frac{\partial^{h} f\left(x^{1}, \ldots, x^{m-1}, t\right)}{\partial x^{1} \partial x^{m^{*}+1} \ldots \partial x^{m}} d t=\frac{\partial^{h-1} f\left(x^{1}, \ldots, x^{m-1}, t_{0}\right)}{\partial x^{1} \partial x^{m^{*}+1} \ldots \partial x^{m-1}}-\frac{\partial^{h-1} f\left(x^{1}, \ldots, x^{m-1}, 0\right)}{\partial x^{1} \partial x^{m^{*}+1} \ldots \partial x^{m-1}} .
$$

From the discussion in $\S 5,2^{\circ}$ we conclude that for almost all $t_{0}$

$$
\int_{a}^{b} \ldots \int_{a}^{b}\left|\frac{\partial^{h-1} f\left(x^{1}, \ldots, x^{m-1}, t_{0}\right)}{\partial x^{1} \partial x^{m+1} \ldots \partial x^{m-1}}\right| d x^{1} \ldots d x^{m-1}<\infty
$$

for all finite values of $a$ and $b .(9.2)$ is valid for almost every line parallel to the $x^{m}$-axis as $C_{\alpha}\left(F_{0}\right)=0, \alpha=m-h$ and $h>1$. Hence we obtain from (9.2) and the assumption on $f$ that (9.3) is true with $t_{0}=0$ for all finite values of $a$ and $b$. If $h=2$ this gives a contradiction to the choices of $F_{0}$ and $f_{0}$. If $h>2$ we repeat the above procedure with (9.1) replaced by the second term of the right member of (9.2). An induction argument finally shows that

$$
\int_{a}^{b} \ldots \int_{a}^{b}\left|\frac{\partial f\left(x^{1}, \ldots, x^{m^{*}}, 0, \ldots, 0\right)}{\partial x^{1}}\right| d x^{1} \ldots d x^{m^{*}}<\infty
$$

for all finite values of $a$ and $b$ which once more gives a contradiction to the choices of $F_{0}$ and $f_{0}$. This proves the theorem.

The type of sets $F_{0}$ used in Example 1 and in the proof of Theorem 6 has a rather complicated structure. As a comparison we consider a case when $F_{0}$ has a simple structure and still satisfies $C_{\alpha}\left(F_{0}\right)=0, \alpha=m-h$.

Example 2. ${ }^{1}$ Consider the case $m=2$ and let $F_{0}$ be the boundary of the open unit circle $U$. Then the following assertions are true:

1 This example is due to Professor L. Carleson. The example is related to results by Gagliardo [12]. 
H. WALLIN, Continuous functions and potential theory

$1^{\circ}$. Every function $f_{0} \in \mathcal{S}\left(F_{0}\right)$ can be extended to a function $f$ which is defined and continuous everywhere in $R^{2}$, infinitely differentiable on $\mathbf{C} F_{0}$, and such that the partial derivatives of the first order of $f$ are in $L^{1}$.

$2^{\circ}$. Let $G$ be a non-decreasing postitve function defined in the interval $r \geqslant 0$ satisfying

$$
\lim _{r \rightarrow \infty} \frac{G(r)}{r}=\infty
$$

Then there exists a function $f_{0} \in S\left(F_{0}\right)$ that cannot be extended to a function $f$ which is continuous in the closure of $U$, infinitely differentiable in $U$ and satisfies

$$
\iint_{U} \mathrm{G}(|\operatorname{grad} f|) d x^{1} d x^{2}<\infty
$$

Proof of $1^{\circ}$. Let $f_{0}$ be a given function from $S\left(F_{0}\right)$. We introduce polar coordinates $(r, \theta)$ and consider $f_{0}$ as a function of $\theta$. We write $f_{0}$ on the following form, where $\varphi_{n}$, for $n=1,2, \ldots$, is an infinitely differentiable function of one variable with period $2 \pi$ and $\max _{0 \leqslant \theta \leqslant 2 \pi}\left|\varphi_{n}(\theta)\right| \leqslant M \cdot 2^{-n}$ for a certain constant $M$,

$$
f_{0}(\theta)=\sum_{1}^{\infty} \varphi_{n}(\theta)
$$

Put $\max _{0 \leqslant \theta \leqslant 2 \pi}\left|\varphi_{n}^{\prime}(\theta)\right|=a_{n}, n=1,2, \ldots$, and choose $\left\{\varepsilon_{n}\right\}, 1>\varepsilon_{n}>0, \lim _{n \rightarrow \infty} \varepsilon_{n}=0$, such that $\sum_{1}^{\infty} \varepsilon_{n} a_{n}<\infty$.

We start by extending $f_{0}$ to $U$. Let $\left\{q_{n}\right\}$ be a sequence of functions defined in the interval $r \geqslant 0$ with $q_{n}$ non-decreasing, infinitely differentiable and such that $q_{n}(r)=0$ if $r \leqslant 1-\varepsilon_{n}$ and $q_{n}(r)=1$ if $r \geqslant 1$. As our extension of $f_{0}$ to $U$ we choose the function $f$ defined by

$$
f\left(x^{1}, x^{2}\right)=\sum_{1}^{\infty} q_{n}(r) \varphi_{n}(\theta), x^{1}=r \cos \theta, x^{2}=r \sin \theta .
$$

$f$ is clearly infinitely differentiable in $U$ and for a certain constant $M_{1}$ we have

$$
\begin{aligned}
\iint_{V}\left(\left|\frac{\partial f}{\partial x^{1}}\right|+\left|\frac{\partial f}{\partial x^{2}}\right|\right) d x^{1} d x^{2} & \leqslant \sum_{n=1}^{\infty} M_{1} \int_{0}^{1} \int_{0}^{2 \pi}\left(\left|q_{n}^{\prime}(r) \varphi_{n}(\theta)\right|+\left|q_{n}(r) \varphi_{n}^{\prime}(\theta)\right|\right) r d \theta d r \\
& \leqslant M_{1} \sum_{1}^{\infty}\left(2 \pi M \cdot 2^{-n}+2 \pi \cdot a_{n} \varepsilon_{n}\right)<\infty
\end{aligned}
$$

i.e. the partial derivatives of the first order of $f$ are absolutely integrable over $U$. Analogously we can, of course, extend $f_{0}$ to the exterior of $U$ with the extended function equal to zero outside a compact set, which proves $1^{\circ}$.

Proof of $2^{\circ}$. Suppose that $f_{0}$ is a function from $S\left(F_{0}\right)$ which can be extended to a function $f$ having the properties stated for the function $f$ in $2^{\circ}$. Let $f(r, \theta)$ 
be the value of $f$ at the point with polar coordinates $(r, \theta)$. If we again consider $f_{0}$ as a function of $\theta$ we have $f(1, \theta)=f_{0}(\theta)$. We get, if $0<\varrho<1$ and $\delta>0$,

$$
\left|f_{0}\left(\theta_{0}+\delta\right)-f_{0}\left(\theta_{0}\right)\right| \leqslant \int_{\varrho}^{1}\left|\frac{\partial f\left(r, \theta_{0}+\delta\right)}{\partial r}\right| d r+\int_{\theta_{0}}^{\theta_{0}+\delta}\left|\frac{\partial f(\varrho, \theta)}{\partial \theta}\right| d \theta+\int_{\varrho}^{1}\left|\frac{\partial f\left(r, \theta_{0}\right)}{\partial r}\right| d r .
$$

Integration over $\theta_{0}$ yields

$$
\begin{aligned}
\int_{0}^{2 \pi}\left|f_{0}\left(\theta_{0}+\delta\right)-f_{0}\left(\theta_{0}\right)\right| d \theta_{0} & \leqslant M_{1} \int_{0}^{2 \pi} \int_{e}^{1}\left|\operatorname{grad} f\left(r, \theta_{0}\right)\right| d r d \theta_{0} \\
& +M_{1} \int_{0}^{2 \pi} d \theta_{0} \int_{\theta_{0}}^{\theta_{0}+\delta}|\operatorname{grad} f(\varrho, \theta)| d \theta=I+I I,
\end{aligned}
$$

where $M_{1}$ is a constant. We divide the domain of integration of $I$ into two parts, one of which consists of those points where $\left|\operatorname{grad} f\left(r, \theta_{0}\right)\right|>(1-\varrho)^{-1 / 2}$. Using (9.4) and (9.5) we obtain, with a constant $M_{2}$ only depending on $f$ and a number $\varepsilon(\varrho)$ only depending on $\varrho, \varepsilon(\varrho) \rightarrow 0, \varrho \rightarrow 1$,

$$
I<M_{2}(\varepsilon(\varrho)+\sqrt{1-\varrho}) .
$$

To estimate $I I$ we observe that

$$
I I=M_{1} \delta \int_{0}^{2 \pi}|\operatorname{grad} f(\varrho, \theta)| \mathrm{d} \theta
$$

and, by (9.5), this quantity is less than $M_{3} \delta(1-\varrho)^{-1}$ for a certain value $\varrho=\varrho_{n}$ where $2^{-n-1} \leqslant 1-\varrho_{n} \leqslant 2^{-n}, n=1,2, \ldots$ Using this and (9.7) it is easy to realize. that we can choose $\varrho$ depending on $\delta$ with $\varrho \rightarrow 1$ when $\delta \rightarrow 0$, so that we obtain a modulus of continuity $t(\delta), t \delta) \rightarrow 0$ when $\delta \rightarrow 0$, for the left member of $(9.6)$, a modulus of continuity which is independent of $f_{0}$ :

$$
\int_{0}^{2 \pi}\left|f_{0}(\theta+\delta)-f_{0}(\theta)\right| d \theta \leqslant M t(\delta), \quad \text { for every } \delta>0 .
$$

$M$ is a constant depending on $f_{0}$ and $f$. Thus (9.8) holds true for all the functions from $S\left(F_{0}\right)$ which can be extended to functions having the properties stated for the function $f$ in $2^{\circ}$. It is, however, not hard to realize that there exists a function $f_{0} \in S\left(F_{0}\right)$ which does not satisfy (9.8).

\section{REFER E N C ES}

1. Besicovxtch, A. S., On lincar sots of points of fractional dimonsion. Math. Ann. 101 (1929), 161-193.

2. Brelot, M., Points irréguliers ot transformations continues en théorio du potontiel. Journal de Math. 19 (1940), 319-337.

3. Carleson, L., On the connoction botwoen Hausdorff moasures and capacity. Arkiv för matematik $B, 3, \mathrm{nr} .36(1957), 403-406$.

4. - Selected problems on exceptional sets. Uppsala (1961), 1-81.

5. Deny, J., Les potenticls d'ónorgio finie. Acta Math. 82 (1950), 107-183. 


\section{H. WALLin, Continuous functions and potential theory}

6. -_._. Sur la convergence de certaines intégrales de la théorie du potentiel. Archiv der Math. 5 (1954), 367-370.

7. Deny, J. and Lions, J. L., Les espaces du type de Beppo Levi. Ann. Inst. Fourier Grenoble, 5 (1955), 305-370.

8. Evans, G. C., Potentials and positively infinite singularities of harmonic functions. Monatshefte für Math. und Phys. 43 (1936), 419-424.

9. Frostman, O., Potentiel d'équilibre et capacité des ensembles avec quelques applications à la théorie des fonctions. Thèse (1935).

10. Fuglede, B., On generalized potentials of functions in the Lebesgue classes. Math. Scand. 8 (1960), 287-304.

11. - On the theory of potentials in locally compact spaces. Acta Math. 103 (1960), 139-215.

12. Gagliardo, E., Caratterizzazioni delle tracce sulla frontiera relative ad alcune classi di funzioni in $n$ variabili. Rend. Sem. Mat. Univ. Padova 27 (1957), 284-305.

13. Kunugui, K., Étude sur la théorie du potentiel généralisé. Osaka Math. J 2 (1950), 63-103.

14. Nikodym, O., Sur une classe de fonctions considérées dans l'étude du problème de Dirichlet. Fund. Math. 21 (1933), 129-150.

15. du Plessis, N., Some theorems about the Riesz fractional integral. Trans. Amer. Math. Soc 80 (1955), $124-134$.

16. Rudin, W., Positive infinities of potentials. Proc. Amer. Math. Soc. 2 (1951), 967-969.

17. Schwartz, L., Théorie des distributions, I-II. Paris (1950-51).

18. UGaFerI, T., On the general potential and capacity. Jap. J. Math. 20 (1950), 37-43. 